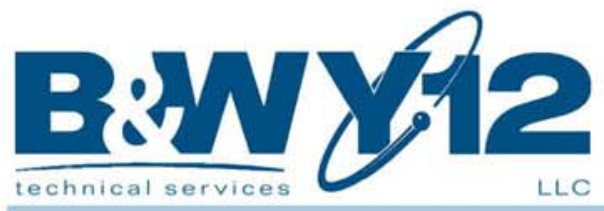

\title{
Y-12 GROUNDWATER PROTECTION PROGRAM GROUNDWATER AND SURFACE WATER SAMPLING AND ANALYSIS PLAN FOR CALENDAR YEAR 2010
}

September 2009

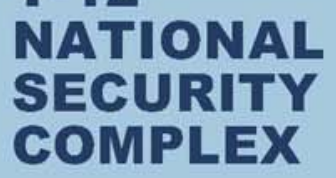

for the

Environmental Compliance Department Environment, Safety, and Health Division Y-12 National Security Complex

Oak Ridge, Tennessee 37831

\section{Managed by}

Babcock \& Wilcox Technical Services Y-12, LLC for the U.S. DEPARTMENT OF ENERGY under contract No. DE-AC05-00OR22800 


\section{DISCLAIMER}

This report was prepared as an account of work sponsored by an agency of the United States Government. Neither the United States Government nor any agency thereof, nor any of their employees, makes any warranty, express or implied, or assumes any legal liability or responsibility for the accuracy, completeness, or usefulness of any information, apparatus, product, or process disclosed, or represents that its use would not infringe privately owned rights. Reference herein to any specific commercial product, process, or service by trade name, trademark, manufacturer, or otherwise, does not necessarily constitute or imply its endorsement, recommendation, or favoring by the United States Government or any agency thereof. The views and opinions of authors expressed herein do not necessarily state or reflect those of the United States Government or any agency thereof. 


\title{
Y-12 GROUNDWATER PROTECTION PROGRAM GROUNDWATER AND SURFACE WATER SAMPLING AND ANALYSIS PLAN FOR CALENDAR YEAR 2010
}

\author{
September 2009
}

\section{Prepared by}

Elvado Environmental LLC Under Subcontract No. 4300068789

\author{
for the \\ Environmental Compliance Department \\ Environment, Safety, and Health Division \\ Y-12 National Security Complex \\ Oak Ridge, Tennessee 37831
}

Managed by

Babcock \& Wilcox Technical Services Y-12, LLC for the U.S. DEPARTMENT OF ENERGY under contract No. DE-AC05-00OR22800 


\section{TABLE OF CONTENTS}

Section

Page

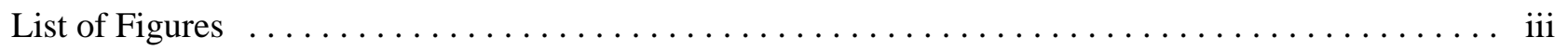

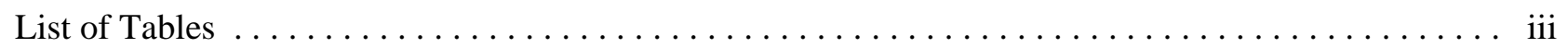

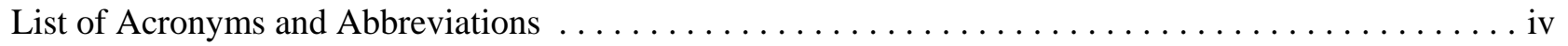

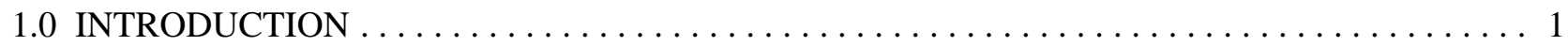

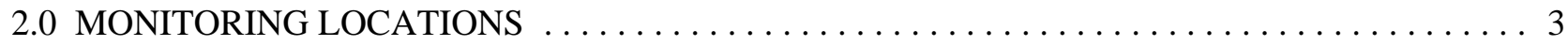

3.0 FIELD MEASUREMENTS AND ANALYTICAL PARAMETERS $\ldots \ldots \ldots \ldots \ldots \ldots \ldots \ldots$

4.0 SAMPLE PLANNING, COLLECTION, AND HANDLING $\ldots \ldots \ldots \ldots \ldots \ldots \ldots \ldots \ldots$

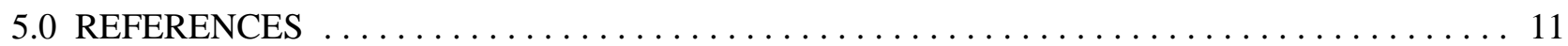

\section{APPENDICES:}

A FIGURES

B TABLES

C CY 2010 GROUNDWATER MONITORING SCHEDULES

D ADDENDA TO THE CY 2010 SAMPLING AND ANALYSIS PLAN

E LABORATORY REQUIREMENTS

(Bottle Lists, Holding Times, Turnaround Times, Elevated Minimum Detectable Activity)

F MANAGEMENT OF PURGED GROUNDWATER 


\section{List of Figures}

$\underline{\text { Figure }}$

$\underline{\text { Page }}$

A.1 Hydrogeologic regimes at the Y-12 National Security Complex $\ldots \ldots \ldots \ldots \ldots \ldots \ldots$ A-1

A.2 CY 2010 sampling locations in the Bear Creek Hydrogeologic Regime $\ldots \ldots \ldots \ldots \ldots \ldots$ A-2

A.3 CY 2010 sampling locations in the Chestnut Ridge Hydrogeologic Regime . . . . . . . . . A A-3

A.4 CY 2010 sampling locations in the Upper East Fork Poplar Creek

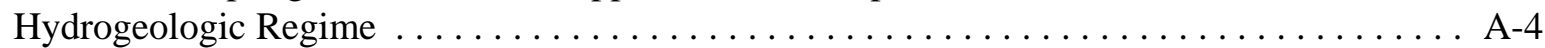

A.5 CY 2010 surface water sampling locations north of Pine Ridge $\ldots \ldots \ldots \ldots \ldots \ldots \ldots$

A.6 Westbay ${ }^{\mathrm{TM}}$ monitoring system sampling port depths in well GW-722 $\ldots \ldots \ldots \ldots \ldots$ A-6

\section{List of Tables}

Table

$\underline{\text { Page }}$

B.1 Sampling locations, frequency, and analytical parameters for groundwater and surface water monitoring during $\mathrm{CY} 2010 \ldots \ldots \ldots \ldots \ldots \ldots \ldots \ldots \ldots \ldots \ldots \ldots \ldots \ldots$

B.2 Field measurements and analytes that comprise the elementary parameter groups for CY 2010 groundwater and surface water samples . . . . . . . . . . . . . . . . . . B B-7 


\section{List of Acronyms and Abbreviations}

ACO

Bear Creek Regime

BWXT

Chestnut Ridge Regime

CY

DOE

East Fork Regime

GWPP

GWMS

PDB

Redox

VOCs

$\mathrm{Y}-12$

\author{
Analytical Chemistry Organization \\ Bear Creek Hydrogeologic Regime \\ BWXT Y-12, L.L.C. \\ Chestnut Ridge Hydrogeologic Regime \\ calendar year \\ U.S. Department of Energy \\ Upper East Fork Poplar Creek Hydrogeologic Regime \\ Groundwater Protection Program \\ Groundwater Monitoring Schedule \\ passive diffusion bag (sampler) \\ oxidation-reduction potential \\ volatile organic compounds \\ Y-12 National Security Complex
}




\subsection{INTRODUCTION}

This plan provides a description of the groundwater and surface water quality monitoring activities planned for calendar year (CY) 2010 at the U.S. Department of Energy (DOE) Y-12 National Security Complex (Y-12) that will be managed by the Y-12 Groundwater Protection Program (GWPP). Groundwater and surface water monitoring performed by the GWPP during CY 2010 will be in accordance with requirements of DOE Order 540.1A and the following goals:

- to protect the worker, the public, and the environment;

- to maintain surveillance of existing and potential groundwater contamination sources;

- to provide for the early detection of groundwater contamination and determine the quality of groundwater and surface water where contaminants are most likely to migrate beyond the Oak Ridge Reservation property line;

- to identify and characterize long-term trends in groundwater quality at Y-12; and

- to provide data to support decisions concerning the management and protection of groundwater resources.

Groundwater and surface water monitoring during CY 2010 will be performed primarily in three hydrogeologic regimes at Y-12: the Bear Creek Hydrogeologic Regime (Bear Creek Regime), the Upper East Fork Poplar Creek Hydrogeologic Regime (East Fork Regime), and the Chestnut Ridge Hydrogeologic Regime (Chestnut Ridge Regime). The Bear Creek and East Fork regimes are located in Bear Creek Valley, and the Chestnut Ridge Regime is located south of Y-12 (Figure A.1). Additional surface water monitoring will be performed north of Pine Ridge, along the boundary of the Oak Ridge Reservation.

Modifications to the CY 2010 monitoring program may be necessary during implementation. Changes in programmatic requirements may alter the analytes specified for selected monitoring wells or may add or remove wells from the planned monitoring network. All modifications to the monitoring program will be approved by the Y-12 GWPP manager and documented as addenda to this sampling and analysis plan.

The following sections of this report provide details regarding the CY 2010 groundwater and surface water monitoring activities. Section 2 describes the monitoring locations in each regime and the processes used to select the sampling locations. A description of the field measurements and laboratory analytes is provided in Section 3. Sample collection methods and procedures are described in Section 4, and Section 5 lists the documents cited for more detailed operational and technical information.

The narrative sections of the report reference several appendices. Figures (maps and diagrams) and tables (excluding data summary tables presented in the narrative sections) are in Appendix A and Appendix B, respectively. Groundwater Monitoring Schedules (when issued throughout CY 2010) will be inserted in Appendix C, and addenda to this plan (if issued) will be inserted in Appendix D. Laboratory requirements (bottle lists, holding times, etc.) are provided in Appendix E, and an approved Waste Management Plan is provided in Appendix F. 


\subsection{MONITORING LOCATIONS}

The monitoring locations to be sampled by the Y-12 GWPP during CY 2010 (Table B.1) were selected based on the sampling frequencies defined in the Y-12 GWPP monitoring optimization plan (Babcock \& Wilcox Technical Services Y-12, LLC 2009). The monitoring wells selected for sample collection in CY 2010 include semiannual, annual, biennial (even years), and pentennial (every five years) sampling frequencies (Table B.1).

The Y-12 GWPP monitoring network for CY 2010 includes 108 monitoring locations (Table B.1): 40 located in the Bear Creek Regime (Figure A.2), nine located in the Chestnut Ridge Regime (Figure A.3), 56 located in the East Fork Regime (Figure A.4), and three located north of Pine Ridge (Figure A.5). Groundwater samples will be collected from a total of 96 monitoring wells, including 36 wells in the Bear Creek Regime (Figure A.2), four wells in the Chestnut Ridge Regime, and 56 wells in the East Fork Regime (Figure A.4). One of the wells located in the East Fork Regime contains a Westbay ${ }^{\mathrm{TM}}$ multiport sampling system that allows collection of groundwater samples from several discrete depth intervals (Table B.1). Well GW-722 will have samples collected from five ports (Figure A.6) during CY 2010. Samples of groundwater discharging from three natural springs will be collected during CY 2010, including spring SS-4 in the Bear Creek Regime (Figure A.2) and springs SCR2.1SP and SCR2.2SP in the Chestnut Ridge Regime (Figure A.3).

Surface water samples will be collected from a total of eight sampling locations during CY 2010, including two locations in the Bear Creek Regime, three locations in the Chestnut Ridge Regime, and three locations north of Pine Ridge. In the Bear Creek Regime, samples will be collected from two stations (BCK-09.40 and BCK-11.97) in the main channel of Bear Creek (Figure A.2). The tributaries located in the Chestnut Ridge Regime have been numbered from west to east (SCR1 through SCR5) and surface water samples will be collected from three of the tributaries at stations (SCR1.5SW, SCR3.5SW, and S17 [located in SCR5]) located along the north side of Bethel Valley Road (Figure A.3). The surface water sampling locations north of Pine Ridge (Figure A.5) include a tributary near the Scarboro Community (NPR12.0SW), a tributary to Mill Branch (NPR23.0SW), and Gum Hollow Branch near Country Club Estates (GHK2.51ESW). 


\subsection{FIELD MEASUREMENTS AND ANALYTICAL PARAMETERS}

Before collecting samples at each monitoring location, field personnel will record (on Field Data Sheets) field measurements (Table B.2), including:

- depth to the static water level in monitoring wells;

- $\mathrm{pH}$;

- water temperature;

- conductivity;

- dissolved oxygen; and

- oxidation-reduction potential (Redox)

Field measurement of dissolved oxygen and Redox will not be obtained for sampling ports of monitoring wells equipped with a Westbay ${ }^{\mathrm{TM}}$ multiport sampling system. Instead of measuring the depth to the static water level in each Westbay ${ }^{\mathrm{TM}}$ sampling zone, the potentiometric head (in $\mathrm{ft}$ ) will be calculated from subsurface pressure measurements obtained.

For this Sampling and Analysis Plan, specific analytes are grouped by analytical method or by type (e.g., trace metals) and referenced as elementary parameter groups (Table B.1 and Table B.2). In addition to field measurements, most of the groundwater and surface water samples will be analyzed for the following suite of parameters (identified as the Standard Administrative Parameter Group):

- miscellaneous laboratory analytes (total suspended solids and total dissolved solids);

- major anions;

- trace metals (includes major cations);

- a comprehensive suite of volatile organic compounds (VOCs); and

- gross alpha and gross beta activity.

Selective parameter monitoring (SPM) will be performed on samples from selected monitoring wells with analytical results for at least eight samples obtained since January 1991. Historical data must clearly demonstrate that the selected parameters are the contaminants of concern and provide sufficient data to characterize the other parameters without additional analyses. For example, samples from 32 monitoring wells will be analyzed only for VOCs (Table B.1), and historical data for these locations show consistently low results for inorganic and radiochemical analytes. The SPM elementary parameter groups reflect analytical methods (Table B.2) and are designed to obtain the data necessary to meet requirements of the GWPP monitoring program.

Samples from selected locations will be analyzed for specific radionuclides. The radionuclide analyses will supplement gross alpha and/or gross beta activity results, especially in cases where the gross activity reporting limits are elevated from interferences caused by a high dissolved solid content of the groundwater sample (see Appendix E). 


\subsection{SAMPLE PLANNING, COLLECTION, AND HANDLING}

The monitoring locations to be sampled during CY 2010 are grouped by hydrogeologic regime to provide geographic areas for planning and tracking purposes. The CY quarter for sample collection at each monitoring location is provided in Table B.1.

A Groundwater Monitoring Schedule(GWMS) will be prepared by GWPP personnel for each sampling event of CY 2010. Each GWMS (four per year) will be issued before sample collection begins, will specify the sequence for collecting samples from the monitoring locations scheduled, and will include information necessary for field personnel to collect the required samples (e.g., containment requirements and previous pumping rates used to sample each well). The GWMS is an integral part of this document, and when issued, the GWMS for each CY 2010 sampling event is to be inserted (Appendix C) by the recipient.

Unfiltered samples will be collected semiannually (22 samples) or annually (101 samples, including 18 biennial and one pentennial sample) from the monitoring locations during CY 2010 (Table B.1). As summarized below, the number of samples to be collected during each CY quarter will range from 19 to 42, for an annual total of 123 samples.

\begin{tabular}{|c|c|c|c|c|}
\hline \multirow{2}{*}{$\begin{array}{c}\text { HYDROGEOLOGIC } \\
\text { REGIME/AREA }\end{array}$} & 1st & 2nd & 3rd & 4th \\
\cline { 2 - 5 } & 16 & 0 & 28 & 0 \\
Bear Creek Regime & 0 & 9 & 0 & 0 \\
Chestnut Ridge Regime & 23 & 14 & 11 & 19 \\
East Fork Regime & 0 & 0 & 3 & 0 \\
North of Pine Ridge & $\mathbf{3 9}$ & $\mathbf{2 3}$ & $\mathbf{4 2}$ & $\mathbf{1 9}$ \\
\hline TotaL: & & & & \\
\hline
\end{tabular}

Personnel from the Environmental Sampling Section of the Y-12 Environment Compliance Department will be responsible for collection, transportation, and chain-of-custody control of all groundwater and surface water samples. Based on the analytical parameters for the CY 2010 monitoring locations (Table B.1 and Table B.2), personnel with the Y-12 Analytical Chemistry Organization (ACO) prepare bottle lists that specify the sample container type, size, preservative, and the laboratory test identification needed for each sampling location (see Appendix E). Additionally, ACO personnel will generate a weekly tracking report to record the sample collection date and time for each monitoring location, the date that analyses are scheduled for completion, or when analyses are completed. Sample collection will be performed in accordance with the most recent version of operating procedures for obtaining groundwater samples (BWXT Y-12, L.L.C. [BWXT] 2002a, BWXT 2004, BWXT 2006b, and BWXT 2007b) and surface water samples (BWXT 2002b). All field and laboratory activities will be performed in accordance with applicable requirements of the Y-12 Integrated Safety Management System and task-specific job hazard analyses.

Groundwater samples will be collected using the low-flow minimal drawdown method (low-flow method) during CY 2010 from most of the monitoring wells (Table B.1). A passive (no purging) sampling method will be used to collect samples at selected monitoring wells either by collecting a sample using the dedicated pump without purging (three wells) or by using a passive diffusion bag (PDB) sampler (24 wells). Additionally, groundwater samples from well GW-722, that is equipped with a Westbay ${ }^{\mathrm{TM}}$ multiport sampling system, will be collected following applicable procedures. 
For the low-flow method, a bladder pump is permanently installed in each well that is scheduled for sample collection. If well construction prevents permanent installation (e.g., flush-mounted wells), then the pump and tubing will be installed at least 24 hours before sample collection and will be removed when sampling is completed. In accordance with the groundwater sampling procedure for the low-flow method (BWXT 2007b), groundwater is purged, and subsequently sampled, from the well at a flow rate ( $<300$ milliliters per minute $[\mathrm{ml} / \mathrm{min}]$ ) which ensures minimal drawdown of the static water level, therefore isolating the stagnant water column above the intake of the pump. Groundwater samples are collected from a well after the water level is in steady-state drawdown $(<0.1 \mathrm{ft}$ over a 15 -minute interval $)$ and field parameters ( $\mathrm{pH}$, conductivity, water temperature, Redox, and dissolved oxygen) have stabilized (minimal variation over four consecutive readings).

A "no-purge method" will be used for wells with low-flow sampling histories that demonstrate very low pumping rate $(<50 \mathrm{ml} / \mathrm{min})$ to meet the minimal drawdown requirement during purging and sample collection. For this method, field measurements will be obtained and groundwater samples will be collected after pumping the stagnant water (calculated volume) from the tubing. During CY 2010, the no-purge method will be used to collect groundwater samples from three wells (GW-065 and GW-623) in the Bear Creek Regime and one well (56-4A) in the East Fork Regime (Table B.1)

Passive diffusion bag samplers will be used to evaluate VOC concentrations at 24 of the wells selected for VOC analyses only, including six wells in the Bear Creek Regime and 18 wells in the East Fork Regime (Table B.1). In addition to meeting the SPM criteria (see Section 3.0) for VOCs only, the selected wells have a monitored interval length of $10 \mathrm{ft}$ or less. A PDB is polyethylene bag (semipermeable membrane) that is filled with deionized water and lowered to the monitored interval of the well. Each PDB will remain in the well for at least two weeks to allow VOC concentrations in the bag to reach equilibrium (passive diffusion) with the surrounding groundwater. After retrieval, sample bottles for VOC analyses will be filled with water from the PDB.

Groundwater sampling and pressure profiling using a Westbay ${ }^{\mathrm{TM}}$ multiport sampling system at well GW-722 (Figure A.6) in the East Fork Regime will be performed in accordance with the applicable operating procedures (BWXT 2002a and BWXT 2006b). The groundwater samples from each sampling port will be collected in 250-milliliter nonvented stainless steel Westbay ${ }^{\mathrm{TM}}$ sample collection bottles filled at the designated depth in the well. Once filled, the bottles will be raised to the surface and the groundwater will be transferred to laboratory sample containers. The sample collection bottles will be lowered, filled, and retrieved as many times as needed to completely fill the laboratory sample bottles. Groundwater in the first sample collection bottles retrieved from each sampling port will be used as a "formation rinse" to obtain field measurements and to condition the sample collection bottle for each zone.

In addition to the groundwater and surface water samples, field blanks and equipment rinsate samples will be collected at the frequencies and analyzed for the parameter groups specified on Table B.1. Field blank samples will be collected for at least $1 \%$ of the samples. Therefore, two field blank samples will be collected during CY 2010: in the Chestnut Regime during the second quarter and in the East Fork Regime during the fourth quarter. An equipment rinsate sample will be collected from Westbay well GW-722 (Table B.1) immediately after field-cleaning the sampling equipment used to collect samples from the last sampling port (GW-722-17).

Trip blank samples and field duplicate samples will be prepared and handled in accordance with the Field Quality Control Samples operating procedure (BWXT 2007c) and will be analyzed using applicable procedures. Trip blank samples will be prepared for each cooler used to transport samples for volatile organic analyses. Because duplicate samples will be collected from at least $10 \%$ of the sampling locations, a total 
of 13 field duplicate samples will be collected during CY 2010: four in the Bear Creek Regime, one in the Chestnut Ridge Regime, four in the East Fork Regime, and one at a surface water station located north of Pine Ridge (Table B.1).

All groundwater and surface water samples will be relinquished under chain-of-custody control to the appropriate Y-12 ACO laboratory that will perform the analyses. The Y-12 ACO laboratories will perform each analyses within established holding times and deliver results in hard copy and electronic format within established turnaround times (see Appendix E). 


\subsection{REFERENCES}

American Public Health Association. 1992. Standard Methods for Examination of Water and Wastewater, $18^{\text {th }}$ Edition.

Babcock \& Wilcox Technical Services Y-12 LLC. 2009. Y-12 Groundwater Protection Program Monitoring Optimization Plan for Groundwater Monitoring Wells at the U.S. Department of Energy Y-12 National Security Complex, Oak Ridge, Tennessee. Prepared by Elvado Environmental LLC (Y/TS2031/R1).

BWXT Y-12, L.L.C. 2002a. Pressure Profiling of Wells Equipped with Westbay ${ }^{\mathrm{TM}}$ Monitoring System Instrumentation. BWXT Y-12, L.L.C. Management Requirement prepared by the Environment, Safety, and Health Organization (Y50-71-019, Rev.1).

BWXT Y-12, L.L.C. 2002b. Liquid Grab Sampling. BWXT Y-12, L.L.C. Management Requirement prepared by the Environment, Safety, and Health Organization (Y50-71-005, Rev. 1.1).

BWXT Y-12, L.L.C. 2004. Measurement of Static Water Level Elevation. BWXT Y-12, L.L.C. Management Requirement prepared by the Environment, Safety, and Health Organization (Y50-71-015, Rev 1.0).

BWXT Y-12, L.L.C. 2005. Assessment of the Groundwater Protection Program, Y-12 National Security Complex, Oak Ridge, Tennessee. Prepared by Groundwater Services, Inc. (Y/TS-1984).

BWXT Y-12, L.L.C. 2006a. Y-12 Groundwater Protection Program Monitoring Optimization Plan for Groundwater Monitoring Wells at the U.S. Department of Energy Y-12 National Security Complex, Oak Ridge, Tennessee. Prepared by Elvado Environmental LLC (Y/TS-2031).

BWXT Y-12, L.L.C. 2006b. Groundwater Sampling of Westbay ${ }^{\mathrm{TM}}$ Monitoring System Instrumented Wells. BWXT Y-12, L.L.C. Management Requirement prepared by the Environment, Safety, and Health Organization (Y50-71-018, Rev.2).

BWXT Y-12, L.L.C. 2007a. Calendar Year 2006 Groundwater Monitoring Report, U.S. Department of Energy Y-12 National Security Complex, Oak Ridge, Tennessee. Prepared by Elvado Environmental LLC (Y/SUB/07-054638/1).

BWXT Y-12, L.L.C. 2007b. Groundwater Sampling. BWXT Y-12, L.L.C. Management Requirement prepared by the Environment, Safety, and Health Organization (Y50-71-016, Rev 2.0).

BWXT Y-12, L.L.C. 2007c. Field Quality Control Samples. BWXT Y-12, L.L.C. Management Requirement prepared by the Environment, Safety, and Health Organization (Y71-66-EC-003, Rev 2.0). 
U.S. Environmental Protection Agency. 1983. Methods for Chemical Analysis of Water and Wastes.

U.S. Environmental Protection Agency. 1996. Test Methods for Evaluating Solid Waste Physical/Chemical Methods. 
APPENDIX A

\section{FIGURES}




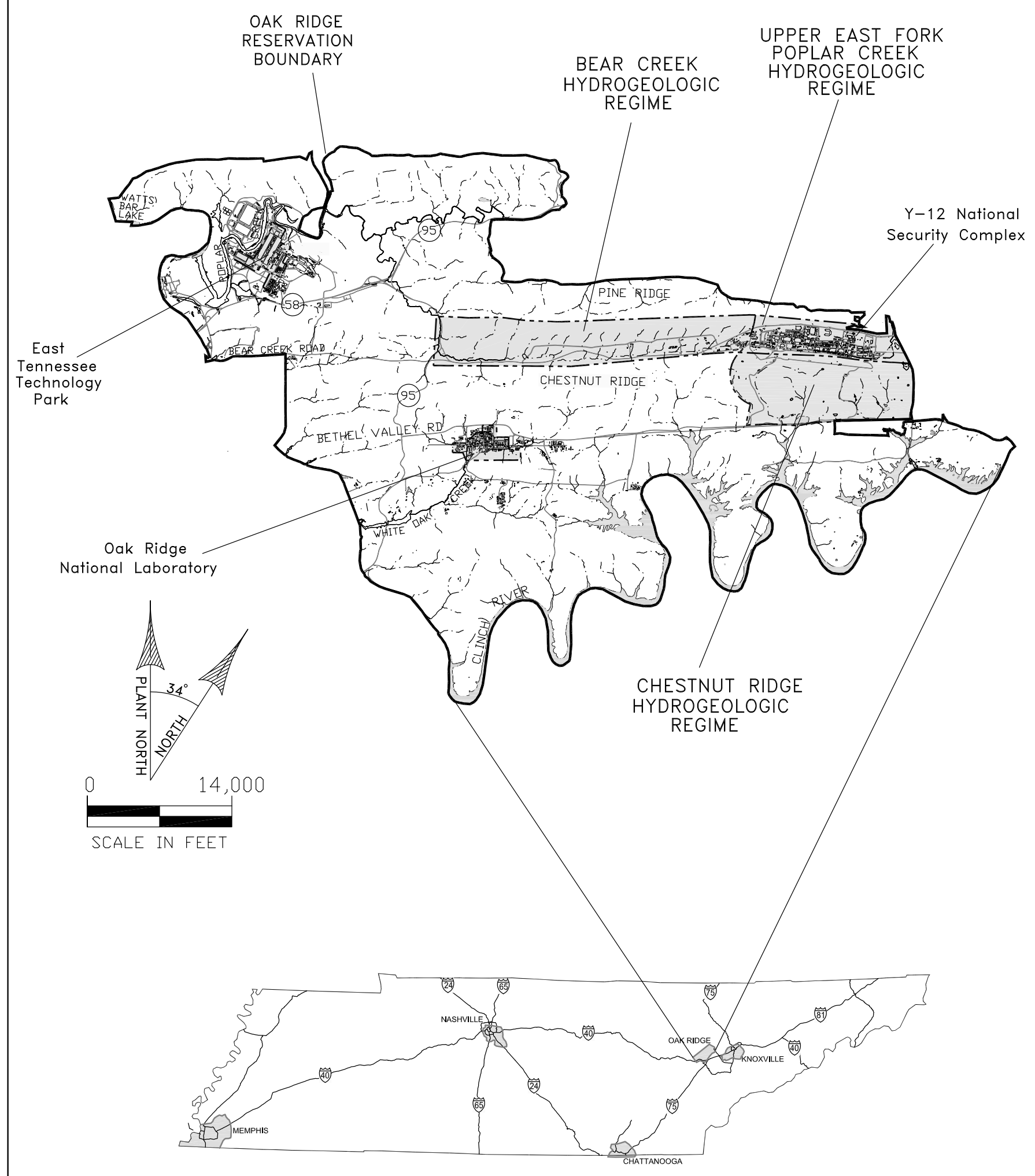

GWPP Fig1 09/23/08

Fig. A.1. Hydrogeologic regimes at the Y-12 National Security Complex. 

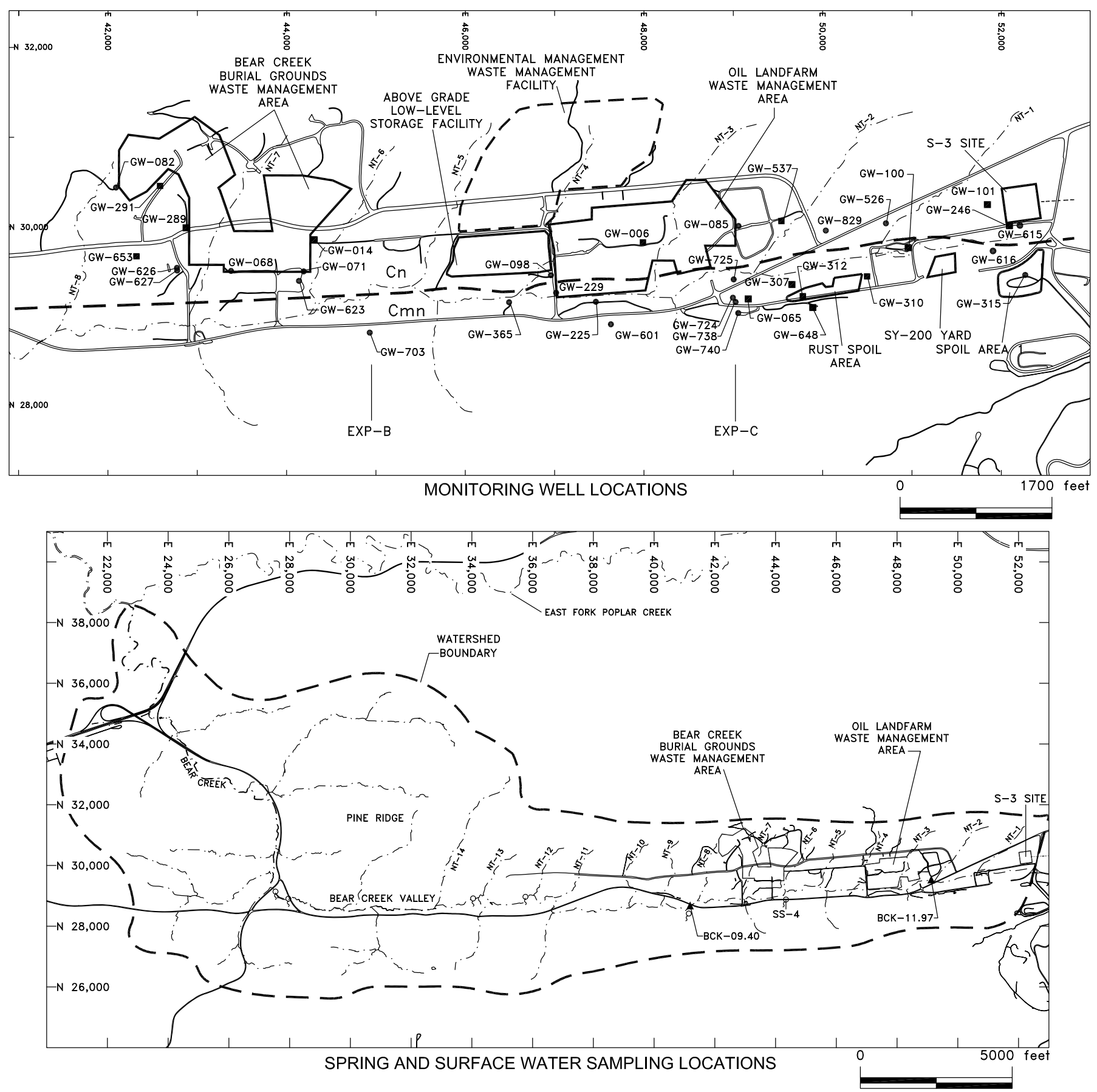

EXPLANATION

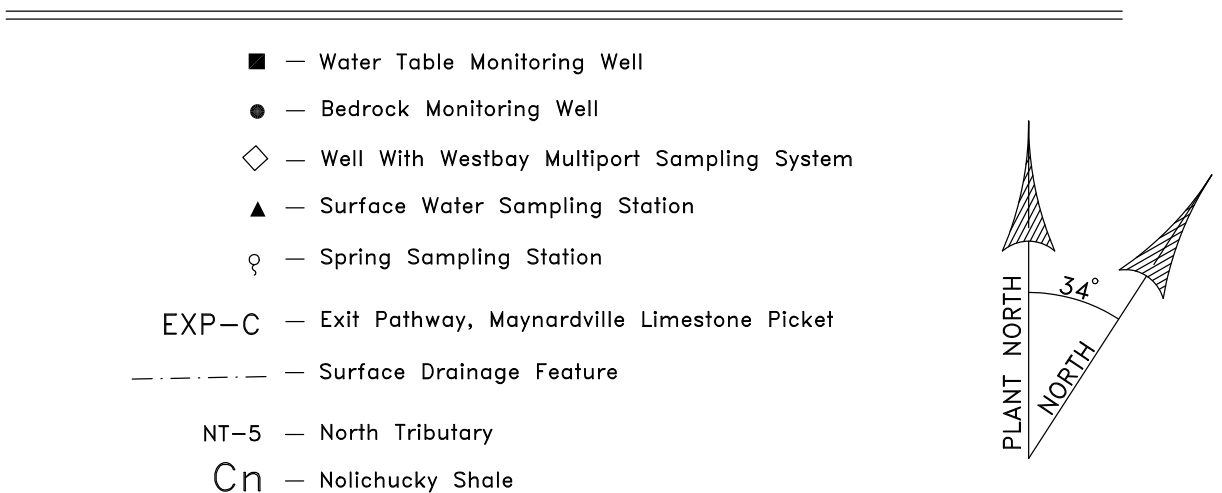

- - - Approximate Geologic Contact

$\mathrm{Cmn}$ - Maynardville Limestone 


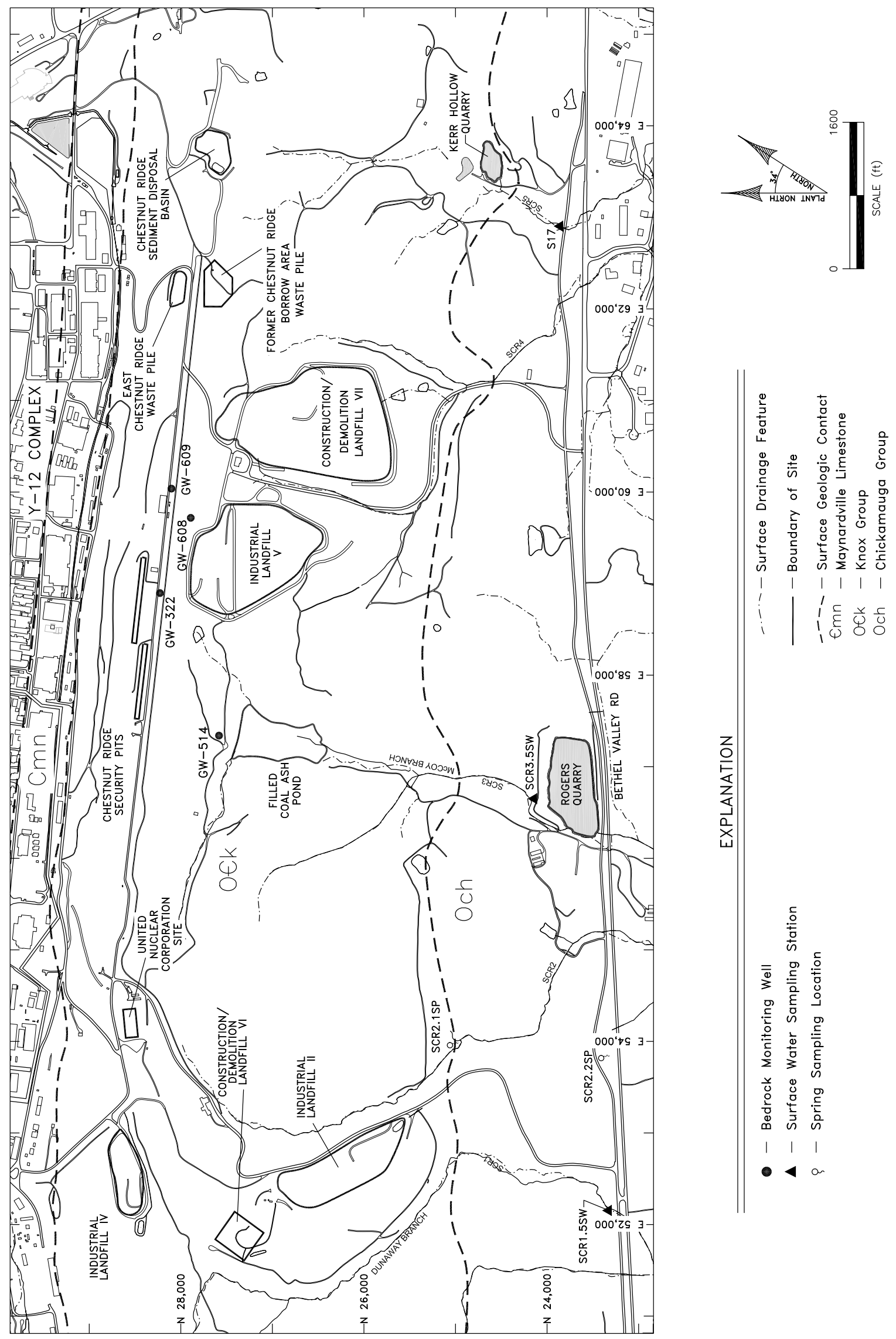

SAP10_3 09/11/09

Fig. A.3. CY 2010 sampling locations in the Chestnut Ridge Hydrogeologic Regime. 


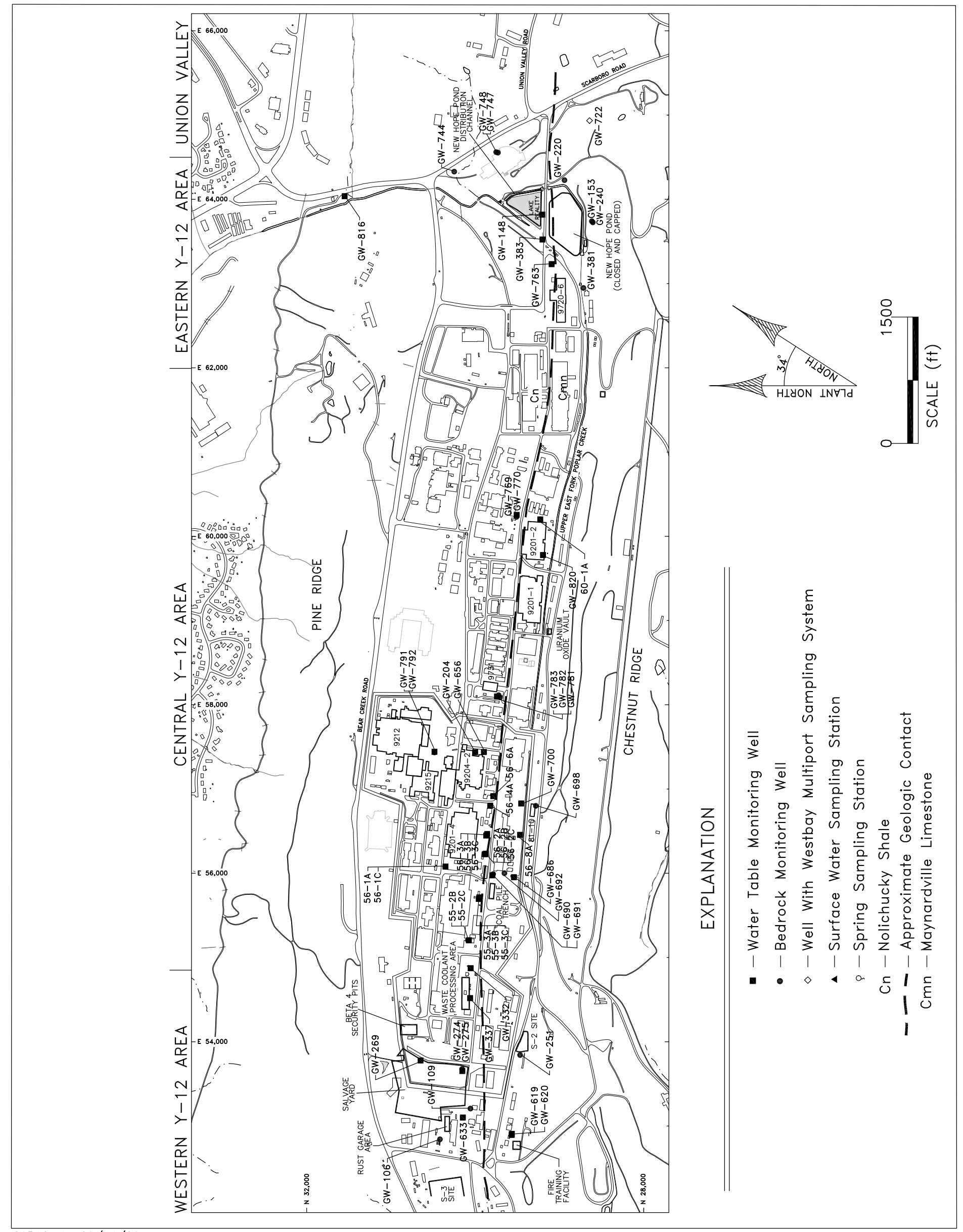

SAP10_4 09/11/09

Fig. A.4. CY 2010 sampling locations in the Upper East Fork Poplar Creek Hydrogeologic Regime. 


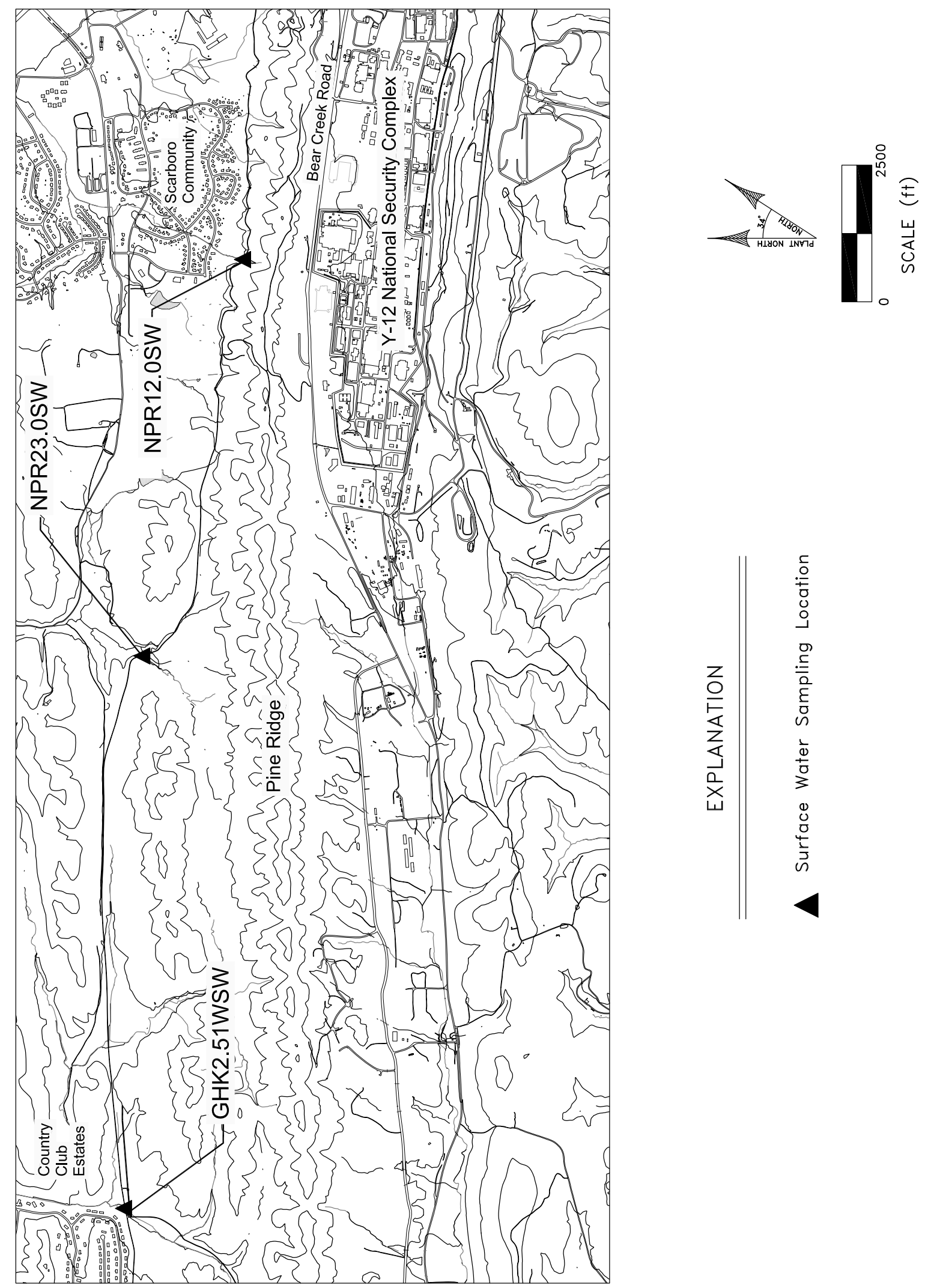




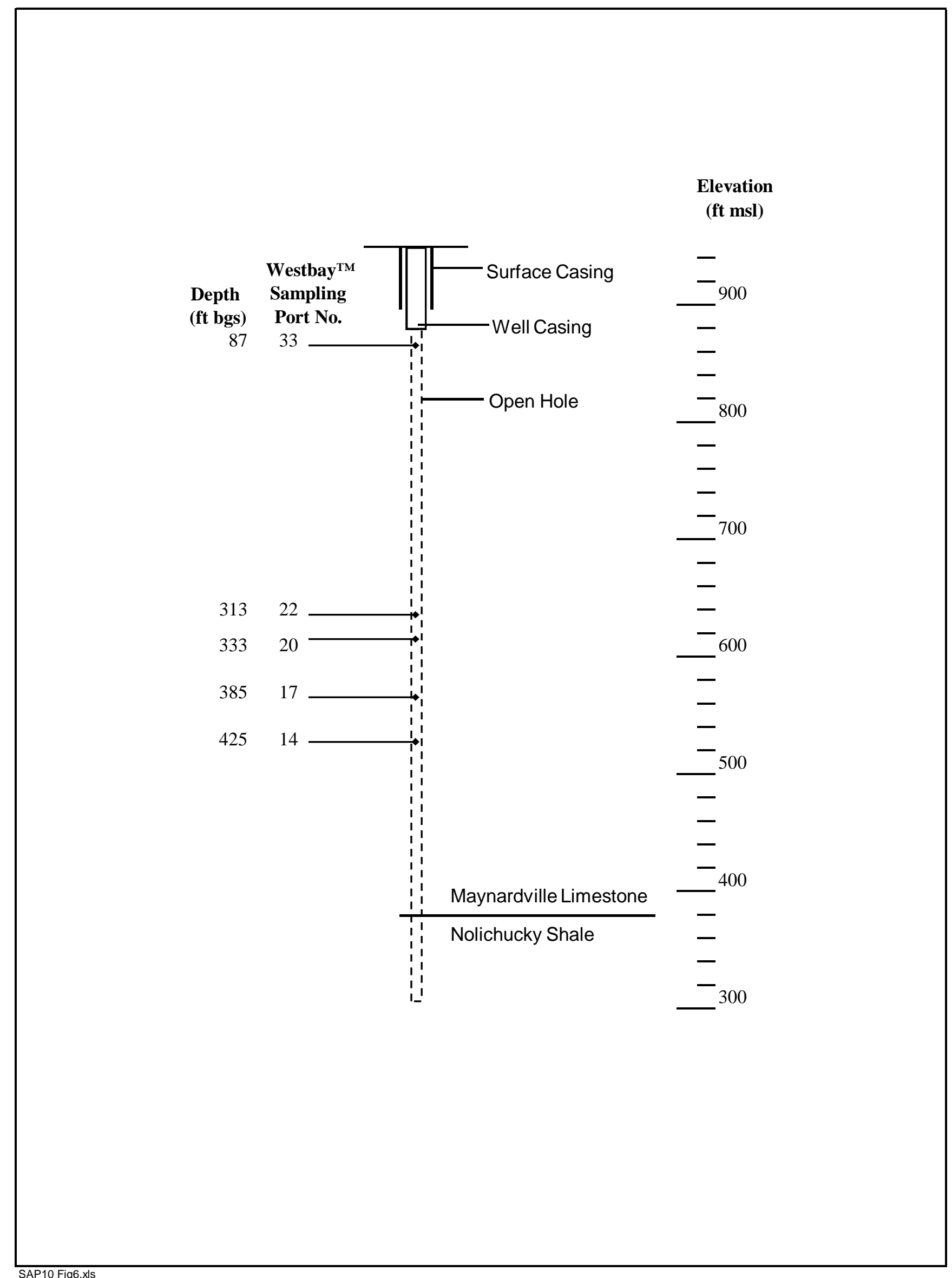

Fig. A.6. Westbay ${ }^{\mathrm{TM}}$ monitoring system sampling port depths in well GW-722. 
APPENDIX B

TABLES 
Table B.1 Sampling locations, frequency, and analytical parameters for groundwater and surface water monitoring during CY 2010

\begin{tabular}{|c|c|c|c|c|c|c|c|c|}
\hline \multirow{2}{*}{$\begin{array}{c}\text { Sampling } \\
\text { Point }^{1} \\
\end{array}$} & \multirow{2}{*}{ Location $^{2}$} & \multirow{2}{*}{$\begin{array}{c}\text { Collection } \\
\text { Method }^{3}\end{array}$} & \multirow{2}{*}{$\begin{array}{c}\text { Sampling } \\
\text { Frequency }\end{array}$} & \multicolumn{4}{|c|}{ Samples Collected in CY $2010^{5}$} & \multirow{2}{*}{ Parameters $^{6}$} \\
\hline & & & & Q1 & Q2 & Q3 & Q4 & \\
\hline \multicolumn{9}{|c|}{ Bear Creek Hydrogeologic Regime } \\
\hline GW-006 & OLF & PDB & Even & Y & & & & VOC-PDB \\
\hline GW-014 & $\mathrm{BG}$ & PDB & Annual & & & Y... & & VOC-PDB \\
\hline GW-065 & OLF & NP & Semiannual & Y & & Y & & STD \\
\hline GW-068 & $\mathrm{BG}$ & "LFLO' & Annual & & & Y" & & STD \\
\hline GW-071 & $\mathrm{BG}$ & LFLO & Semiannual & D & & Y" & & MET-PMS,VOC(1) \\
\hline GW-082 & $\mathrm{BG}$ & LFLO & Annual & Y & & & & VOC(1) \\
\hline GW-085 & OLF & LFLO & Annual & & & Y & & Anions,RAD $(1,12)$ \\
\hline GW-098" & OLF & LFLO" & Annual & & & Y... & & VOC(1) \\
\hline GW-100 & S3 & LFLO & Annual & & & Y & & STD \\
\hline GW-101 & S3 & LFLO" & Annual & & & Y' & & STD \\
\hline GW-225" & OLF" & LFLO" & Annual & & & Y." & & Anions, VOC(1) \\
\hline GW-229 & OLF & LFLO & Annual & & & Y & & STD \\
\hline GW-246 & $\mathrm{S} 3$ & LFLO & Annual & Y... & & & & STD \\
\hline GW-289 & $\mathrm{BG}$ & PDB & Annual & Y... & & & & VOC-PDB \\
\hline GW-291 & $\mathrm{BG}$ & LFLO & Pentennial & & & D & & STD \\
\hline GW-307 & $\mathrm{RS}$ & LFLO & Annual & & & Y & & STD \\
\hline GW-310 & $\mathrm{RS}$ & LFLO & Even & & & Y" & & STD \\
\hline GW-312 & $\mathrm{RS}$ & LFLO" & Even & & & Y & & STD \\
\hline GW-315 & SPI & PDB & Annual & & & Y" & & VOC-PDB \\
\hline GW-365 & OLF & LFLO & Annual & & & Y & & VOC(1) \\
\hline GW-526" & S3 & LFLO" & Annual & & & 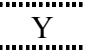 & & Anions,RAD $(1,12)$ \\
\hline GW-537 & OLF & "LFLO" & Annual & Y & & & & Anions,RAD(1,12) \\
\hline GW-601 & OLF" & LFLO" & Annual & & & Y & & Anions, VOC(1) \\
\hline GW-615 & S3 & LFLO & Annual & & & $Y$ & & STD \\
\hline GW-616 & S3 & LFLO & Annual & $\mathrm{Y}$ & & & & Anions \\
\hline GW-623 & $\mathrm{BG}$ & NP & Annual & & & Y... & & VOC(1) \\
\hline GW-626 & $\mathrm{BG}$ & LFLO & Even & Y & & & & STD \\
\hline GW-627 & $\mathrm{BG}$ & LFLO & Semiannual & Y & & Y & & VOC(1) \\
\hline GW-648 & $\mathrm{RS}$ & $\mathrm{PDB}$ & Semiannual & Y" & & Y & & VOC-PDB \\
\hline GW-653 & BG & PDB & Annual & & & Y... & & VOC-PDB \\
\hline GW-703 & EXP-B & LFLO & Annual & & & D & & STD \\
\hline GW-724 & EXP-C & LFLO & Annual & & & 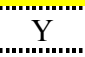 & & Anions, VOC(1) \\
\hline GW-725 & EXP-C & LFEO" & (nnnual & & & 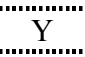 & & Anions,MET-PMS,VOC(1) \\
\hline GW-738 & EXP-C & "LFLO" & Annual & & & Y & & STD \\
\hline GW-740" & EXP-C & LFLO" & Annual & & & Y & & $\operatorname{VOC}(1)$ \\
\hline GW-829 & OLF & LFLO & Even & Y........ & & & & Anions \\
\hline BCK-09.40 & EXP-SW & GRAB & Annual & Y & & & & Anions,MET-PMS \\
\hline BCK-11.97 & EXP-SW & GRAB & Annual & Y & & & & Anions,MET-PMS \\
\hline SS-4 & EXP-SW & GRAB & Annual & D & & & & STD \\
\hline \multicolumn{9}{|c|}{ Chestnut Ridge Hydrogeologic Regime } \\
\hline GW-322 & CRSP & LFLO & Annual & & D & & & $\operatorname{VOC}(1)$ \\
\hline FB-GW-322 & CRSP & & & & Y & & & VOC(1) \\
\hline GW-514 & FCAP & LFLO & Even & & $Y$ & & & STD \\
\hline GW-608 & CRSP & LFLO & Even & & Y & & & STD \\
\hline GW-609 & CRSP & LFLO & Even & & Y & & & STD \\
\hline
\end{tabular}


Table B.1 (continued)

\begin{tabular}{|c|c|c|c|c|c|c|c|c|}
\hline \multirow{2}{*}{$\begin{array}{c}\text { Sampling } \\
\text { Point }^{1}\end{array}$} & \multirow{2}{*}{ Location $^{2}$} & \multirow{2}{*}{$\begin{array}{c}\text { Collection } \\
\text { Method }^{3}\end{array}$} & \multirow{2}{*}{$\begin{array}{c}\text { Sampling } \\
\text { Frequency }\end{array}$} & \multicolumn{4}{|c|}{ Samples Collected in CY $2010^{5}$} & \multirow{2}{*}{ Parameters $^{6}$} \\
\hline & & & & Q1 & Q2 & Q3 & Q4 & \\
\hline \multicolumn{9}{|c|}{ Chestnut Ridge Hydrogeologic Regime (continued) } \\
\hline S17 & EXP-SW & GRAB & Annual & & Y & & & STD \\
\hline SCR1.5SW" & EXP-SW & GRAB & Annual & & Y & & & STD \\
\hline SCR2.1SP' & EXP-SW' & GRAB' & Annual & & Y & & & STD \\
\hline SCR2.2SP & EXP-SW" & GRAB & Annual & & Y's & & & STD" \\
\hline SCR3.5SW & EXP-SW & GRAB & Annual & & $\mathrm{Y}$ & & & STD \\
\hline \multicolumn{9}{|c|}{ Upper East Fork Poplar Creek Hydrogeologic Regime } \\
\hline $55-2 \mathrm{~B}$ & GRIDB3 & LFLO & Annual & & Y & & & Anions, VOC(1) \\
\hline $55-2 \mathrm{C}$ & GRIDB3 & LFLO & Annual & & D & & & Anions, VOC(1) \\
\hline $55-3 \mathrm{~A}$ & B9201-5 & LFLO & Semiannual & Y... & & Y & & STD \\
\hline $55-3 \mathrm{~B}$ & B9201-5 & LFLO" & Semiannual & Y & & Y" & & STD \\
\hline $55-3 C$ & B9201-5 & LFLO & Semiannual & Y & & Y & & STD \\
\hline $56-1 A$ & Y12 & LFLO & Annual & $Y$ & & & & STD \\
\hline $56-1 C$ & Y12 & LFLO & Annual & $Y$ & & & & STD \\
\hline $56-2 A$ & GRIDC3 & PDB & Annual & Y... & & & & VOC-PDB \\
\hline $56-2 B$ & GRIDC3 & PDB & Annual & Y & & & & VOC-PDB \\
\hline $56-2 C$ & GRIDC3 & PDB" & Annual & Y... & & & & VOC-PDB \\
\hline $56-3 A$ & Y12 & LFLO" & Annual & Y & & & & STD \\
\hline $56-3 \mathrm{~B}$ & Y12 & LFLO & Annual & Y.. & & & & STD \\
\hline $56-3 C$ & Y12 & LFLO & Annual & Y... & & & & STD \\
\hline $56-4 \mathrm{~A}$ & Y12 & NP & Annual & Y & & & & STD \\
\hline $56-6 \mathrm{~A}$ & Y12 & LFLO & Even & & & & Y & STD \\
\hline $56-8 \mathrm{~A}$ & Y12 & LFLO & Even & 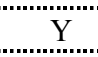 & & & & STD \\
\hline $60-1 \mathrm{~A}$ & Y12 & LFLO & Even & & Y... & & & STD \\
\hline GW-106 & S3 & LFLO & Even & & & & $Y$ & STD \\
\hline GW-109 & $\mathrm{S} 3$ & LFLO & Even & & & & Y & STD \\
\hline GW-148 & NHP & LFLO" & Even & D & & & & STD \\
\hline GW-153 & NHP & PDB & Annual & & Y & & & VOC-PDB \\
\hline GW-204" & T0134 & LFLO" & Annual & & & & Y" & MET-PMS,RAD $(1,3$ \\
\hline GW-220 & NHP & "LFLO" & Semiannual & Y & & Y & & VOC(1) \\
\hline GW-240 & NHP & $\mathrm{PDB}$ & Annual & & Y & & & VOC-PDB \\
\hline GW-251 & $\mathrm{S} 2$ & LFLO & Annual & & & & D & Anions, VOC(1) \\
\hline GW-269 & SY & PDB & Annual & & & & Y & VOC-PDB \\
\hline $\mathrm{GW}-274$ & SY & LFLO & Annual & & & & Y.' & STD \\
\hline GW-275 & SY & LFLO & Annual & & & & Y... & STD \\
\hline GW-332 & WCPA & PDB & Annual & & & & Y... & VOC-PDB \\
\hline FB-GW-332 & WCPA & & & & & & Y.' & VOC(1) \\
\hline GW-337 & WCPA & LFLO & Annual & & & & Y & Anions, VOC(1) \\
\hline GW-381 & NHP & PDB & Annual & & Y & & & VOC-PDB \\
\hline GW-383 & " NHP' & PDB & Annual & & Y & & & VOC-PDB" \\
\hline GW-619" & FTF & PDB & Even & & D & & & VOC-PDB \\
\hline GW-620 & FTF & PDB & Even & & Y & & & VOC-PDB \\
\hline GW-633 & $\mathrm{RG}$ & LFLO & Annual & & & & Y & STD \\
\hline GW-656 & T0134 & LFLO & Annual & & & & Y & STD \\
\hline GW-686 & $\mathrm{CPT}$ & LFLO & Annual & & & & Y & Anions,HG,VOC(1) \\
\hline GW-690 & CPT & LFLO & Annual & & & & Y & Anions, HG, VOC(1) \\
\hline GW-691 & СРT & LFLO & Semiannual & & Y & & Y & Anions,HG,VOC(1) \\
\hline GW-692 & СРT & LFLO & Annual & & & & D & Anions,HG,VOC(1) \\
\hline
\end{tabular}


Table B.1 (continued)

\begin{tabular}{|c|c|c|c|c|c|c|c|c|}
\hline \multirow{2}{*}{$\begin{array}{c}\text { Sampling } \\
\text { Point }^{1}\end{array}$} & \multirow{2}{*}{ Location $^{2}$} & \multirow{2}{*}{$\begin{array}{l}\text { Collection } \\
\text { Method }^{3}\end{array}$} & \multirow{2}{*}{$\begin{array}{c}\text { Sampling } \\
\text { Frequency }\end{array}$} & \multicolumn{4}{|c|}{ Samples Collected in CY $2010^{5}$} & \multirow{2}{*}{ Parameters $^{6}$} \\
\hline & & & & Q1 & Q2 & Q3 & Q4 & \\
\hline \multicolumn{9}{|c|}{ Upper East Fork Poplar Creek Hydrogeologic Regime (continued) } \\
\hline GW-698 & B8110 & LFLO & Semiannual & & Y & & Y & Anions,HG,VOC(1) \\
\hline GW-700 & B 8110 & PDB & Annual & D & & & & VOC-PDB \\
\hline GW-722-14 & EXP-J & WBAY & Annual & & & $\mathrm{Y}$ & & STD \\
\hline GW-722-17 & EXP-J & WBAY & Annual & & & $\mathrm{Y}$ & & STD \\
\hline ER-GW-722-17 & EXP-J & & & & & Y & & VOC $(1)$ \\
\hline GW-722-20 & EXP-J & WBAY & Annual & & & Y & & STD \\
\hline GW-722-22 & EXP-J & WBAY & Annual & & & Y & & STD \\
\hline GW-722-33 & EXP-J & WBAY" & Annual & & & Y... & & STD \\
\hline GW-744 & GRIDK1 & LFLO & Annual & & & 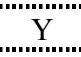 & & STD \\
\hline GW-747 & GRIDK2 & LFLO & Annual & & & 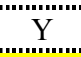 & & STD \\
\hline GW-748 & GRIDK2 & LFLO & Even & & & D & & STD \\
\hline GW-763 & GRIDJ3 & PDB & Annual & & Y & & & VOC-PDB \\
\hline GW-769 & GRIDG3 & PDB & Semiannual & & Y & & Y & VOC-PDB \\
\hline GW-770 & GRIDG3 & PDB & Annual & & Y... & & & VOC-PDB \\
\hline GW-781 & GRIDE3 & LFLO & Annual & Y... & & & & STD \\
\hline GW-782 & GRIDE3 & LFLO & Annual & Y & & & & STD \\
\hline GW-783 & GRIDE3 & PDB & Annual & $\mathrm{Y}$ & & & & VOC-PDB \\
\hline GW-791 & GRIDD2 & PDB & Annual & Y & & & & VOC-PDB \\
\hline GW-792 & GRIDD2 & LFLO & Even & Y & & & & STD \\
\hline GW-816 & EXP-SR & LFLO & Annual & Y" & & & & STD \\
\hline GW-820 & B9201-2 & PDB & Annual & & & & Y & VOC-PDB \\
\hline GHK2.51WSW & "EXP-SW & GRAB' & Annual & & & D & & STD \\
\hline NPR12.0SW" & EXP-SW & GRAB & Annual & & & $Y$ & & STD \\
\hline NPR23.0SW & EXP-SW & GRAB & Annual & & & Y & & STD \\
\hline
\end{tabular}




\section{Table B.1 (continued)}

Notes:

1 BCK - Bear Creek Kilometer (surface water station)

ER - Equipment rinsate sample

FB - Field blank sample

GW - Groundwater monitoring well

GHK - Gum Hollow Kilometer (surface water station), North of Pine Ridge

NPR - North of Pine Ridge (surface water station)

S17 - Surface water station in SCR5

SCR - South Chestnut Ridge (spring or surface water station)

SS - Spring sampling location: South Side of Bear Creek

2 B8110 - Building 81-10

B9201-2 - Building 9201-2

B9201-5 - Building 9201-5

BG - Bear Creek Burial Grounds Waste Management Area

CPT - Coal Pile Trench

CRSP - Chestnut Ridge Security Pits

EXP-B - Exit Pathway Picket B

EXP-C - Exit Pathway Picket C

EXP-J - Maynardville Limestone Exit Pathway Picket J

EXP-SR - Exit pathway well in the gap through Pine Ridge along Scarboro Road

EXP-SW - Spring or Surface Water Location

FCAP - Filled Coal Ash Pond

FTF - Fire Training Facility

GRID - Comprehensive Groundwater Monitoring Plan Grid Location

NHP - New Hope Pond

OLF - Oil Landfarm Waste Management Area

RG - Rust Garage Area

RS - Rust Spoil Area

S2 - S-2 Site

S3 - S3 Site

SPI - Spoil Area I

SY - Y-12 Salvage Yard

T0134 - Underground Storage Tank 0134-U

WCPA - Waste Coolant Processing Area

Y12 - Y-12 Complex

3 Collection Method

LFLO - Low-flow minimal drawdown method

NP - No purge before sample collection; history of very low sampling rate $(<50 \mathrm{ml} / \mathrm{min})$

PDB - Passive diffusion bag

GRAB - Surface water sample, grab sample

WBAY - Westbay multiport method

4 Sampling Frequency:

As described in the Y-12 GWPP monitoring optimization plan

(Babcock \& Wilcox Technical Services Y-12, LLC 2009) 


\title{
Table B.1 (continued)
}

\section{Notes: (continued)}

5 Groundwater Monitoring Schedules (Appendix C) provide the sequence for collecting samples during each quarterly sampling event and include the waste stream identification for groundwater purged from each monitoring well. The Waste Management Plan for sampling activities is in Appendix F.

Y - Sample collection will be performed during the CY 2010 quarter

D - A field duplicate sample will collected in addition to the regular sample

6 Table B.2 provides a comprehensive list of analytes, analytical methods, and the associated parameter group.

STD - Standard administrative parameter group, including all of the analytes in the following elementary parameter groups:

FLD - Field measurements

CHEM - Miscellaneous laboratory analytes (e.g., dissolved solids) and anions

MET(1) - Metals

VOC(1) - Volatile organic compounds

RAD(1) - Gross alpha and gross beta activity

Selective Parameter Monitoring: (Field measurements will be obtained at all locations)

\author{
Anions - Chloride, Nitrate, and Sulfate \\ HG - Mercury \\ MET-ICP - Metals by method SW846-6010B \\ MET-PMS - Metals by method SW846-6020 \\ VOC-PDB - Volatile organic compounds reported for Passive Diffusion Bag samples; \\ a subset of the VOC(1) group \\ $\operatorname{RAD}(3)$ - Uranium-234, -235, and -238 \\ RAD(12) - Technetium-99
}


Table B.2 Field measurements and laboratory analytes that comprise the elementary parameter groups for CY 2010 groundwater and surface water samples

\begin{tabular}{|c|c|c|c|c|c|}
\hline \multicolumn{2}{|c|}{$\begin{array}{c}\text { Parameter } \\
\text { Group }{ }^{1}\end{array}$} & $\begin{array}{c}\text { Measurement or } \\
\text { Analyte }\end{array}$ & $\begin{array}{l}\text { Analytical } \\
\text { Method }^{2}\end{array}$ & $\begin{array}{l}\text { Reporting } \\
\text { Limit }^{3}\end{array}$ & Units $^{4}$ \\
\hline \multirow{6}{*}{\multicolumn{2}{|c|}{ FLD }} & Depth to Water & NA & NA & $\mathrm{ft}$ \\
\hline & & Water Temperature & NÄ & NA" & centigrade \\
\hline & & $\mathrm{pH}$ & NA & NA & pH units \\
\hline & & Conductivity & NA & NA & $\mu \mathrm{mho} / \mathrm{cm}$ \\
\hline & & Dissolved Oxygen & NÄ" & "NA" & ppm \\
\hline & & Oxidation-Reduction Potential (Redox) & NA & NA & $\mathrm{mV}$ \\
\hline \multirow[t]{8}{*}{ CHEM } & TDS & Total Dissolved Solids & SM 2540C 18 & 1 & $\mathrm{mg} / \mathrm{L}$ \\
\hline & TSS & Total Suspended Solids & SM 2540D 18 & 1 & $\mathrm{mg} / \mathrm{L}$ \\
\hline & Alkalinity & Bicarbonate & SM 2320B 18 & 1 & $\mathrm{mg} / \mathrm{L}$ \\
\hline & & Carbonate & SM $2320 \mathrm{~B} 18$ & 1 & $\mathrm{mg} / \mathrm{L}$ \\
\hline & Anions & Chloride & SW846-9056 & 0.2 & $\mathrm{mg} / \mathrm{L}$ \\
\hline & & Nitrate (as Nitrogen) & EPA-353.2 & 0.05 & $\mathrm{mg} / \mathrm{L}$ \\
\hline & & Sulfate & SW846-9056 & 0.25 & $\mathrm{mg} / \mathrm{L}$ \\
\hline & Fluoride & Fluoride & SM $4500 \mathrm{~F} 18$ & 0.1 & $\mathrm{mg} / \mathrm{L}$ \\
\hline \multirow[t]{29}{*}{ MET(1) } & MET-ICP & Aluminum & SW846-6010B & 0.2 & $\mathrm{mg} / \mathrm{L}$ \\
\hline & & Barium & SW846-6010B & 0.004 & $\mathrm{mg} / \mathrm{L}$ \\
\hline & & Beryllium & SW846-6010B & 0.0005 & $\mathrm{mg} / \mathrm{L}$ \\
\hline & & Boron & SW846-6010B & 0.1 & $\mathrm{mg} / \mathrm{L}$ \\
\hline & & Calcium & SW846-6010B & 0.2 & $\mathrm{mg} / \mathrm{L}$ \\
\hline & & Cobait & SW846-6010B & 0.02 & $\mathrm{mg} / \mathrm{L}$ \\
\hline & & Copper & SW846-6010B & 0.02 & mg/L \\
\hline & & Iron & SW846-6010B & 0.05 & $\mathrm{mg} / \mathrm{L}$ \\
\hline & & Lithium & SW846-6010B" & 0.01 & $\mathrm{mg} / \mathrm{L}$ \\
\hline & & Magnesium & SW846-6010B & 0.2 & $\mathrm{mg} / \mathrm{L}$ \\
\hline & & Manganese & SW846-6010B & 0.005 & $\mathrm{mg} / \mathrm{L}$ \\
\hline & & Molybdenum & SW846-6010B & 0.05 & $\mathrm{mg} / \mathrm{L}$ \\
\hline & & Potassium & SW846-6010B & 2 & $\mathrm{mg} / \mathrm{L}$ \\
\hline & & Silver & SW846-6010B & 0.02 & $\mathrm{mg} / \mathrm{L}$ \\
\hline & & Sodium & SW846-6010B & 0.2 & $\mathrm{mg} / \mathrm{L}$ \\
\hline & & Strontium & SW846-6010B & 0.005 & $\mathrm{mg} / \mathrm{L}$ \\
\hline & & Thorium & SW846-6010B & 0.2 & $\mathrm{mg} / \mathrm{L}$ \\
\hline & & Vanadium & SW846-6010B & 0.02 & mg/L \\
\hline & & Zinc & SW846-6010B & 0.05 & $\mathrm{mg} / \mathrm{L}$ \\
\hline & MET-PMS & Antimony & SW846-6020 & 0.0025 & $\mathrm{mg} / \mathrm{L}$ \\
\hline & & Arsenic & SW846-6020 & 0.005 & $\mathrm{mg} / \mathrm{L}$ \\
\hline & & Cadmium & SW846-6020 & 0.0025 & $\mathrm{mg} / \mathrm{L}$ \\
\hline & & Chromium & SW846-6020 & 0.01 & $\mathrm{mg} / \mathrm{L}$ \\
\hline & & Lead & SW846-6020 & 0.0005 & $\mathrm{mg} / \mathrm{L}$ \\
\hline & & Nickel[ & SW846-6020 & 0.005 & $\mathrm{mg} / \mathrm{L}$ \\
\hline & & Selenium & SW846-6020 & 0.01 & $\mathrm{mg} / \mathrm{L}$ \\
\hline & & Thallium & SW846-6020 & 0.0005 & mg/L"' \\
\hline & & Uranium & SW846-6020 & 0.0005 & $\mathrm{mg} / \mathrm{L}$ \\
\hline & $\mathrm{HG}$ & Mercury & SW846-7470A & 0.00005 & $\mathrm{mg} / \mathrm{L}$ \\
\hline \multirow[t]{9}{*}{ VOC(1) } & & Acetone & SW846-8260B-UP & 10 & $\mu \mathrm{g} / \mathrm{L}$ \\
\hline & & Acetonitrile & SW846-8260B-UP & 5 & $\mu \mathrm{g} / \mathrm{L}$ \\
\hline & & Acrolein & SW846-8260B-UP & 10 & $\mu \mathrm{g} / \mathrm{L}$ \\
\hline & & Acrylonitrile & SW846-8260B-UP & 5 & $\mu \mathrm{g} / \mathrm{L}$ \\
\hline & VOC-PDB & Benzene & SW846-8260B-UP & 5 & $\mu \mathrm{g} / \mathrm{L}$ \\
\hline & & Bromochloromethane & SW846-8260B-UP & 5 & $\mu \mathrm{g} / \mathrm{L}$ \\
\hline & VOC-PDB & Bromodichloromethane & SW846-8260B-UP & 5 & 年 \\
\hline & VOC-PDB & Bromoform| & SW846-8260B-UP & 5 & $\mu \mathrm{g} / \mathrm{L}$ \\
\hline & & Bromomethane & SW846-8260B-UP & 5 & $\mu \mathrm{g} / \mathrm{L}$ \\
\hline
\end{tabular}


Table B.2 (continued)

\begin{tabular}{|c|c|c|c|c|c|}
\hline \multicolumn{2}{|c|}{\begin{tabular}{|c|} 
Parameter \\
Group $^{1}$ \\
\end{tabular}} & $\begin{array}{c}\text { Measurement or } \\
\text { Analyte }\end{array}$ & $\begin{array}{l}\text { Analytical } \\
\text { Method }^{2}\end{array}$ & $\begin{array}{c}\text { Reporting } \\
\text { Limit }^{3}\end{array}$ & Units $^{4}$ \\
\hline \multirow{46}{*}{\begin{tabular}{|c|} 
VOC(1) \\
(continued)
\end{tabular}} & \multirow{3}{*}{$\begin{array}{l}\text { VOC-PDB } \\
\text { VOC-PDB }\end{array}$} & 2-Butanone & SW846-8260B-UP & 5 & $\mu \mathrm{g} / \mathrm{L}$ \\
\hline & & Carbon disulfide & SW846-8260B-UP & 5 & $\mu \mathrm{g} / \mathrm{L}$ \\
\hline & & Carbon tetrachloride & SW846-8260B-UP & 5 & $\mu \mathrm{g} / \mathrm{L}$ \\
\hline & VOC-PDB & Chlorobenzene & SW846-8260B-UP & 5 & $\mu \mathrm{g} / \mathrm{L}$ \\
\hline & VOC-PDB & Chloroethane & SW846-8260B-UP & 5 & $\mu \mathrm{g} / \mathrm{L}$ \\
\hline & VOC-PDB & 2-Chloroethylvinyl ether & SW846-8260B-UP & 10 & $\mu \mathrm{g} / \mathrm{L}$ \\
\hline & VOC-PDB & Chloroform & SW846-8260B-UP & 5 & $\mu \mathrm{g} / \mathrm{L}$ \\
\hline & VOC-PDB & Chloromethane & SW846-8260B-UP & 5 & $\mu \mathrm{g} / \mathrm{L}$ \\
\hline & \multirow[t]{3}{*}{ VOC-PDB } & Dibromochloromethane & SW846-8260B-UP & 5 & $\mu \mathrm{g} / \mathrm{L}$ \\
\hline & & 1,2-Dibromo-3-chloropropane & SW846-8260B-UP & 10 & $\mu \mathrm{g} / \mathrm{L}$ \\
\hline & & 1,2-Dibromoethane & SW846-8260B-UP & 5 & $\mu \mathrm{g} / \mathrm{L}$ \\
\hline & VOC-PDB & Dibromomethane & SW846-8260B-UP & 5 & $\mu \mathrm{g} / \mathrm{L}$ \\
\hline & VOC-PDB & 1,2-Dichlorobenzene & SW846-8260B-UP & 5 & $\mu \mathrm{g} / \mathrm{L}$ \\
\hline & \multirow[t]{3}{*}{ VOC-PDB } & 1,4-Dichlorobenzene & SW846-8260B-UP & 5 & $\mu \mathrm{g} / \mathrm{L}$ \\
\hline & & 1,4-Dichloro-2-butene & SW846-8260B-UP & 5 & $\mu \mathrm{g} / \mathrm{L}$ \\
\hline & & trans-1,4-Dichloro-2-butene & SW846-8260B-UP & 5 & $\mu \mathrm{g} / \mathrm{L}$ \\
\hline & VOC-PDB & Dichlorodifluoromethane & SW846-8260B-UP & 5 & $\mu \mathrm{g} / \mathrm{L}$ \\
\hline & VOC-PDB & 1,1-Dichloroettane & SW846-8260B-UP & 5 & $\mu \mathrm{g} / \mathrm{L}$ \\
\hline & VOC-PDB & 1,2-Dichloroethane & SW846-8260B-UP & 5 & $\mu \mathrm{g} / \mathrm{L}$ \\
\hline & VOC-PDB & 1,1-Dichloroethene & SW846-8260B-UP & 5 & $\mu \mathrm{g} / \mathrm{L}$ \\
\hline & VOC-PDB & cis-1,2-Dichloroethene & SW846-8260B-UP & 5 & $\mu \mathrm{g} / \mathrm{L}$ \\
\hline & VOC-PDB & trans-1,2-Dichloroethene & SW846-8260B-UP & 5 & $\mu \mathrm{g} / \mathrm{L}$ \\
\hline & VOC-PDB & 1,2-Dichloropropane & SW846-8260B-UP & & $\mu \mathrm{g} / \mathrm{L}$ \\
\hline & VOC-PDB & cis-1,3-Dichloropropene & SW846-8260B-UP & & $\mu \mathrm{g} / \mathrm{L}$ \\
\hline & \multirow[t]{2}{*}{ VOC-PDB } & trans-1,3-Dichloropropene & SW846-8260B-UP & 5 & $\mu \mathrm{g} / \mathrm{L}$ \\
\hline & & Ethanol| & SW846-8260B-UP & 200 & $\mu \mathrm{g} / \mathrm{L}$ \\
\hline & \multirow[t]{5}{*}{ VOC-PDB } & Ethylbenzene & SW846-8260B-UP & 5 & $\mu \mathrm{g} / \mathrm{L}$ \\
\hline & & Ethyl methacrylate & SW846-8260B-UP & 5 & $\mu \mathrm{g} / \mathrm{L}$ \\
\hline & & 2-Hexanone & SW846-8260B-UP & & $\mu \mathrm{g} / \mathrm{L}$ \\
\hline & & Iodomethane & SW846-8260B-UP & 5 & $\mu \mathrm{g} / \mathrm{L}$ \\
\hline & & 4-Methyl-2-pentanone & SW846-8260B-UP & 5 & $\mu \mathrm{g} / \mathrm{L}$ \\
\hline & \multirow[t]{3}{*}{ VOC-PDB } & Methylene chloride & SW846-8260B-UP & 5 & $\mu \mathrm{g} / \mathrm{L}$ \\
\hline & & Styrene & SW846-8260B-UP & 5 & $\mu \mathrm{g} / \mathrm{L}$ \\
\hline & & $1,1,1,2-$ Tetrachloroethane & SW846-8260B-UP & 5 & $\mu \mathrm{g} / \mathrm{L}$ \\
\hline & VOC-PDB & $1,1,2,2-$ Tetrachloroethane & SW846-8260B-UP & 5 & $\mu \mathrm{g} / \mathrm{L}$ \\
\hline & VOC-PDB & Tetrachloroethene & SW846-8260B-UP & 5 & $\mu \mathrm{g} / \mathrm{L}$ \\
\hline & VOC-PDB & Toluene & SW846-8260B-UP & 5 & $\mu \mathrm{g} / \mathrm{L}$ \\
\hline & VOC-PDB & Total Xylene & SW846-8260B-UP & 5 & $\mu \mathrm{g} / \mathrm{L}$ \\
\hline & VOC-PDB & $1,1,1$-Trichloroethane & SW846-8260B-UP & 5 & $\mu \mathrm{g} / \mathrm{L}$ \\
\hline & VOC-PDB & $1,1,2$-Trichloroethane & SW846-8260B-UP & 5 & $\mu \mathrm{g} / \mathrm{L}$ \\
\hline & VOC-PDB & Trichloroethene & SW846-8260B-UP & 5 & $\mu \mathrm{g} / \mathrm{L}$ \\
\hline & VOC-PDB & Trichlorofluoromethane & SW846-8260B-UP & 5 & $\mu \mathrm{g} / \mathrm{L}$ \\
\hline & VOC-PDB & $1,2,3$-Trichloropropane & SW846-8260B-UP & 10 & $\mu \mathrm{g} / \mathrm{L}$ \\
\hline & \multirow[t]{3}{*}{ VOC-PDB } & 1,1,2-Trichloro-1,2,2-trifluoroethane & SW846-8260B-UP & 5 & $\mu \mathrm{g} / \mathrm{L}$ \\
\hline & & Vinyl acetate & SW846-8260B-UP & 10 & $\mu \mathrm{g} / \mathrm{L}$ \\
\hline & & Vinyl chloride & SW846-8260B-UP & 2 & $\mu \mathrm{g} / \mathrm{L}$ \\
\hline \multirow{2}{*}{\multicolumn{2}{|c|}{ RAD(1) }} & Gross Alpha Activity & EPA-900.0 & 5 & $\mathrm{pCi} / \mathrm{L}$ \\
\hline & & Gross Beta Activity & EPA-900.0 & 10 & $\mathrm{pCi} / \mathrm{L}$ \\
\hline \multicolumn{2}{|l|}{ RAD(3) } & Uranium-234, -235, \& -238 & Y50-AC-65-7061 & 0.4 & $\mathrm{pCi} / \mathrm{L}$ \\
\hline \multicolumn{2}{|l|}{ RAD(12) } & Technetium-99 & Y $50-A C-65-7060$ & 15 & $\mathrm{pCi} / \mathrm{L}$ \\
\hline
\end{tabular}


Table B.2 (continued)

Notes:

1 Elementary Parameter Groups for the Standard Parameter Group and Selected Parameter Monitoring:

FLD - Field measurements

CHEM - Miscellaneous laboratory analytes (e.g., dissolved solids) and anions

Anions - Chloride, Nitrate, and Sulfate

MET(1) - All Metals (MET-ICP, HG, and MET-PMS)

MET-ICP - Metals by method SW846-6010B (19 metals)

HG - Mercury

MET-PMS - Metals by method SW846-6020 (10 metals)

VOC(1) - Volatile organic compounds (54 compounds)

VOC-PDB - Volatile organic compounds reported for Passive Diffusion Bag samples (36 compounds)

$\mathrm{RAD}(1)$ - Gross alpha and gross beta activity

$\mathrm{RAD}(3)$ - Uranium-234, -235, and -238

RAD(12) - Technetium-99

2 Analytical Method:

NA - Not Applicable

Field measurements are performed in accordance with the following B\&W Y-12

Management Requirements operating procedures:

\begin{tabular}{|cl|cc|}
\hline Field Measurement & Procedure & Field Measurement & Procedure \\
\hline Depth to Water & Y50-71-015 & Dissolved Oxygen & Y50-71-032 \\
Water Temperature & Y50-71-030,-014 & Redox & Y50-71-033 \\
pH & Y50-71-031,-014 & Pressure Profile & Y50-71-019 \\
Conductivity & Y50-71-034,-022 & & \\
\hline
\end{tabular}

Analytical methods from:

- $\quad$ EPA - Methods for Chemical Analysis of Water and Wastes

(U.S. Environmental Protection Agency 1983)

- $\quad$ SM - Standard Methods for the Evaluation of Water and Wastewater, 18th Edition (American Public Health Association 1992)

- $\quad$ SW846 - Test Methods for Evaluating Solid Waste Physical/Chemical Methods (U.S. Environmental Protection Agency 1996)

- $\quad$ B\&W Y-12 ACO Procedures and laboratory test names on the table below are applicable to the analytical methods shown above in the main table:

\begin{tabular}{|cll|}
\hline Method & ACO Procedure & ACO Lab Test \\
\hline EPA-353.2 & ASO-TP-7659 & NO3-N \\
EPA-900.0 & Y50-AC-65-7074 & GROSSAB-ENV \\
SM 2320B 18 & Y/P65-7639 & ALKALINITY-I \\
SM 2540C 18 & Y-50-AC-65-7914 & SOLIDS-TOT-D \\
SM 2540D 18 & Y/P65-7918 & SOLIDS-TOT-S \\
SM 4500F 18 & Y/P65-7602 & FLUORIDE \\
SW846-6010B & Y50-AC-65-0040 & ICP6010 \\
SW846-6020 & Y/P65-0034 & ICPMSGW \\
SW846-7470A & Y50-AC-65-7470 & HGLOWRL \\
SW846-8260B-UP & Y/P65-SW846-8260B & VOA8260GW \\
SW846-8260B-UP & Y/P65-SW846-8260B & VOAGW-PDB \\
SW846-9056 & Y50-AC-65-7619 & ANIONS \\
Y50-AC-65-7060 & Y50-AC-65-7060 & TC99LS-ENV \\
Y50-AC-65-7061 & Y50-AC-65-7061 & ASPECU-ENV \\
\hline
\end{tabular}


Table B.2 (continued)

\section{Notes: (continued)}

3 Reporting Limits:

NA - not applicable

VOC - Reporting limits are contract-required quantitation limits; also report estimated values (with qualifier) below this limit and above the method detection limit.

RAD - Reporting limits are target minimum detectable activities (MDAs) that may be obtained under optimal analytical conditions; actual MDAs are sample-specific and may vary significantly from the target value.

4 Units:

$\mathrm{mg} / \mathrm{L} \quad$ - milligrams per liter

$\mathrm{mV}$ - millivolts

NTU - nephelometric turbidity units

ppm - parts per million

$\mathrm{pCi} / \mathrm{L}$ - picoCuries per liter 
APPENDIX C

CY 2009 GROUNDWATER MONITORING SCHEDULES (Insert When Issued, Before Each Quarterly Sampling Event) 
APPENDIX D

ADDENDA TO THE CY 2009 SAMPLING AND ANALYSIS PLAN (if issued) 
APPENDIX E

LABORATORY REQUIREMENTS

(Bottle Lists, Holding Times, Turnaround Time, Elevated Minimum Activity) 


\section{CY 2010 SAP BOTTLE LISTS}

\section{STD}

\begin{tabular}{|c|c|c|c|}
\hline Parameter & Lab Tests & $\begin{array}{c}\text { Chemical } \\
\text { Preservative }{ }^{1}\end{array}$ & Bottle Types/Size \\
\hline $\begin{array}{l}\text { Anions, Fluoride, } \\
\text { Carbonate and } \\
\text { Bicarbonate }\end{array}$ & $\begin{array}{l}\text { ANIONS, } \\
\text { FLUORIDE, } \\
\text { ALKALINITY-I }\end{array}$ & None & 1 - $250 \mathrm{~mL}$ polyethylene \\
\hline Nitrate & NO3-N & $\begin{array}{c}\mathrm{H}_{2} \mathrm{SO}_{4} \text { to } \mathrm{pH}< \\
2 ; 4^{\mathrm{O}}+1-2^{\mathrm{O}}\end{array}$ & 1 - $100 \mathrm{~mL}$ polyethylene \\
\hline Total Suspended Solids & SOLIDS-TOT-S & None & 1 - $250 \mathrm{~mL}$ polyethylene \\
\hline Total Dissolved Solids & SOLIDS-TOT-D & None & 1-250 mL polyethylene \\
\hline $\begin{array}{l}\text { Total Metals (ICP,ICP- } \\
\text { MS, and } \mathrm{Hg} \text { ) }\end{array}$ & $\begin{array}{l}\text { ICP6010, } \\
\text { ICPMS6020-EXT, } \\
\text { HGLOWRL }\end{array}$ & $\mathrm{HNO}_{3}$ & $1-500 \mathrm{~mL}$ polyethylene \\
\hline Radiochemistry (UV) & GROSSAB-ENV & $\mathrm{HNO}_{3}$ & 1-1 L polyethylene \\
\hline Volatiles & VOA8260GW & None & $\begin{array}{l}2-40 \mathrm{~mL} \text { amber glass } \\
\text { with Teflon lined septum } \\
\text { lids }\end{array}$ \\
\hline $\begin{array}{l}\text { Trip Blank (VOA) } \\
\text { (one per cooler) }\end{array}$ & VOA8260GW & None & $\begin{array}{l}1-40 \mathrm{~mL} \text { amber glass } \\
\text { with Teflon lined septum } \\
\text { lid }\end{array}$ \\
\hline
\end{tabular}

STD: $\quad$ LIMS LAB TEST ID

CHEM ALKALINITY-I, ANIONS, NO3-N, FLUORIDE, SOLIDS-TOT-S, SOLIDS-TOT-D

MET(1) ICP6010, ICPMS6020-EXT and HGLOWRL

VOC(1) VOA8260GW

RAD(1)GROSSAB-ENV

${ }^{1}$ All samples chilled to $4^{\circ} \mathrm{C}+/-2^{\circ} \mathrm{C}$ 


\section{CY 2010 SAP BOTTLE LISTS}

Anions, RAD (1)

\begin{tabular}{||l|c|c|c||}
\hline \multicolumn{1}{|c|}{ Parameter } & Lab Tests & $\begin{array}{c}\text { Chemical } \\
\text { Preservative }\end{array}$ & Bottle Types/Size \\
\hline \hline Anions & ANIONS & None & $1-250 \mathrm{~mL}$ polyethylene \\
\hline Nitrate & $\mathrm{NO}-\mathrm{N}$ & $\begin{array}{c}\mathrm{H}_{2} \mathrm{SO}_{4} \mathrm{to} \mathrm{pH}_{2 ;}< \\
24^{\mathrm{O}}+/-2^{\mathrm{O}}\end{array}$ & $1-100 \mathrm{~mL}$ polyethylene \\
\hline Gross Alpha/Beta & GROSSAB-ENV & $\mathrm{HNO}_{3}$ & $1-1$ L polyethylene \\
\hline
\end{tabular}

\section{Parameters:}

Anions

Nitrate

$\mathrm{RAD}(1)$

${ }^{1}$ All samples chilled to $4{ }^{\circ} \mathrm{C}+/-2^{\circ} \mathrm{C}$

\section{LIMS LAB TEST ID}

ANIONS

NO3-N

GROSSAB-ENV 


\section{CY 2010 SAP BOTTLE LISTS}

\section{STD-WESTBAY}

\begin{tabular}{|c|c|c|c|}
\hline Parameter & Lab Tests & $\begin{array}{c}\text { Chemical } \\
\text { Preservative }^{1}\end{array}$ & Bottle Types/Size \\
\hline $\begin{array}{l}\text { Anions, Fluoride, } \\
\text { Carbonate and } \\
\text { Bicarbonate }\end{array}$ & $\begin{array}{l}\text { ANIONS, } \\
\text { FLUORIDE, } \\
\text { ALKALINITY-I }\end{array}$ & None & 1 - $250 \mathrm{~mL}$ polyethylene \\
\hline Nitrate & NO3-N & $\begin{array}{c}\mathrm{H}_{2} \mathrm{SO}_{4} \text { to } \mathrm{pH}< \\
2 ; 4^{\mathrm{O}}+1-2^{\mathrm{O}}\end{array}$ & $1-100 \mathrm{~mL}$ polyethylene \\
\hline Total Suspended Solids & SOLIDS-TOT-S & None & 1 - $250 \mathrm{~mL}$ polyethylene \\
\hline Total Dissolved Solids & SOLIDS-TOT-D & None & 1 - $250 \mathrm{~mL}$ polyethylene \\
\hline $\begin{array}{l}\text { Total Metals (ICP,ICP- } \\
\mathrm{MS} \text {, and } \mathrm{Hg} \text { ) }\end{array}$ & $\begin{array}{l}\text { ICP6010, } \\
\text { ICPMS6020-EXT, } \\
\text { HGLOWRL }\end{array}$ & $\mathrm{HNO}_{3}$ & $1-250 \mathrm{~mL}$ polyethylene \\
\hline Radiochemistry (UV) & GROSSAB-ENV & $\mathrm{HNO}_{3}$ & $\begin{array}{l}1-500 \mathrm{~mL} \\
\text { polyethylene }\end{array}$ \\
\hline Volatiles & VOA8260GW & None & $\begin{array}{l}2-40 \mathrm{~mL} \text { amber glass } \\
\text { with Teflon lined septum } \\
\text { lids }\end{array}$ \\
\hline $\begin{array}{l}\text { Trip Blank (VOA) } \\
\text { (one per cooler) }\end{array}$ & VOA8260GW & None & $\begin{array}{l}1-40 \mathrm{~mL} \text { amber glass } \\
\text { with Teflon lined septum } \\
\text { lid }\end{array}$ \\
\hline
\end{tabular}

\section{STD: $\quad$ LIMS LAB TEST ID}

CHEM ALKALINITY-I, ANIONS, NO3-N, FLUORIDE, SOLIDS-TOT-S, SOLIDS-TOT-D MET(1) ICP6010, ICPMS6020-EXT and HGLOWRL

VOC(1) VOA8260GW

RAD(1)GROSSAB-ENV

${ }^{1}$ All samples chilled to $4{ }^{\circ} \mathrm{C}+/-2^{\circ} \mathrm{C}$ 


\section{CY 2010 SAP BOTTLE LISTS}

\section{Anions}

\begin{tabular}{||l|c|c|c||}
\hline \multicolumn{1}{|c|}{ Parameter } & Lab Tests & $\begin{array}{c}\text { Chemical } \\
\text { Preservative }\end{array}$ & Bottle Types/Size \\
\hline \hline Anions & ANIONS & None & $1-250 \mathrm{~mL}$ polyethylene \\
\hline \hline Nitrate & $\mathrm{NO}-\mathrm{N}$ & $\begin{array}{c}\mathrm{H}_{2} \mathrm{SO}_{4} \text { to } \mathrm{pH}< \\
2 ; 4^{\mathrm{O}}+/ 2^{\mathrm{O}}\end{array}$ & $1-100 \mathrm{~mL}$ polyethylene \\
\hline
\end{tabular}

Parameter:

Anions

Nitrate
LIMS LAB TEST ID

ANIONS

NO3-N

${ }^{1} \mathrm{All}$ samples chilled to $4^{\circ} \mathrm{C}+/-2^{\circ} \mathrm{C}$ 


\section{CY 2010 SAP BOTTLE LISTS}

\section{Anions, VOC (1)}

\begin{tabular}{||l|c|c|c||}
\hline \multicolumn{1}{|c|}{ Parameter } & Lab Tests & $\begin{array}{c}\text { Chemical } \\
\text { Preservative }\end{array}$ & Bottle Types/Size \\
\hline \hline Anions & ANIONS & None & $1-250 \mathrm{~mL}$ polyethylene \\
\hline \hline Nitrate & NO3-N & $\begin{array}{c}\mathrm{H}_{2} \mathrm{SO}_{4} \text { to } \mathrm{pH}< \\
2 ; 4^{\mathrm{O}}+/ 2^{\mathrm{O}}\end{array}$ & $1-100 \mathrm{~mL}$ polyethylene \\
\hline \hline Volatiles & VOA8260GW & None & $\begin{array}{l}2-40 \mathrm{~mL} \text { amber glass } \\
\text { with Teflon lined septum } \\
\text { lids }\end{array}$ \\
\hline
\end{tabular}

Parameter:

Anions

Nitrate

$\operatorname{vOC}(1)$

\section{LIMS LAB TEST ID}

ANIONS

NO3-N

VOA8260GW

${ }^{1}$ All samples chilled to $4^{\circ} \mathrm{C}+/-2^{\circ} \mathrm{C}$ 


\section{CY 2010 SAP BOTTLE LISTS}

\section{Anions, HG, VOC (1)}

\begin{tabular}{||l|c|c|c||}
\hline \multicolumn{1}{|c|}{ Parameter } & Lab Tests & $\begin{array}{c}\text { Chemical } \\
\text { Preservative }\end{array}$ & \multicolumn{1}{|c||}{ Bottle Types/Size } \\
\hline \hline Anions & ANIONS & None & $1-250 \mathrm{~mL}$ polyethylene \\
\hline \hline Nitrate & NO3-N & $\begin{array}{c}\mathrm{H}_{2} \mathrm{SO}_{4} \text { to } \mathrm{pH}< \\
2 ; 4^{\mathrm{O}}+/-2^{\mathrm{O}}\end{array}$ & $1-100 \mathrm{~mL}$ polyethylene \\
\hline \hline Mercury $(\mathrm{Hg})$ & HGLOWRL & HNO3 & $1-250 \mathrm{~mL}$ polyethylene \\
\hline \hline Volatiles & VOA8260GW & None & $\begin{array}{l}2-40 \mathrm{~mL} \text { amber glass } \\
\text { with Teflon lined septum } \\
\text { lids }\end{array}$ \\
\hline
\end{tabular}

Parameter:

Anions

$\operatorname{VOC}(1)$
LIMS LAB TEST ID

ANIONS

VOA8260GW

${ }^{1}$ All samples chilled to $4^{\circ} \mathrm{C}+/-2^{\circ} \mathrm{C}$ 


\section{CY 2010 SAP BOTTLE LISTS}

\section{$\operatorname{VOC}(1)$}

\begin{tabular}{||c|c|c|c||}
\hline \multicolumn{1}{|c|}{ Parameter } & Lab Tests & $\begin{array}{c}\text { Chemical } \\
\text { Preservative }^{1}\end{array}$ & \multicolumn{1}{|c||}{ Bottle Types/Size } \\
\hline \hline Volatiles & VOA8260GW & None & $\begin{array}{l}2-40 \mathrm{~mL} \text { amber glass } \\
\text { with Teflon lined septum } \\
\text { lids }\end{array}$ \\
\hline
\end{tabular}

Parameter: $\quad$ LIMS LAB TEST ID

VOC(1) VOA8260GW

${ }^{1}$ All samples chilled to $4^{\circ} \mathrm{C}+/-2^{\circ} \mathrm{C}$ 


\section{CY 2010 SAP BOTTLE LISTS}

\section{VOC-PDB}

\begin{tabular}{||l|c|c|c||}
\hline \multicolumn{1}{|c|}{ Parameter } & Lab Tests & $\begin{array}{c}\text { Chemical } \\
\text { Preservative }\end{array}$ & Bottle Types/Size \\
\hline \hline Volatiles & VOA8260GW & None & $\begin{array}{l}2-40 \mathrm{~mL} \text { amber glass } \\
\text { with Teflon lined septum } \\
\text { lids }\end{array}$ \\
\hline
\end{tabular}

Parameter: $\quad$ LIMS LAB TEST ID

VOC-PDB VOAGW-PDB

${ }^{1}$ All samples chilled to $4^{\circ} \mathrm{C}+/-2^{\circ} \mathrm{C}$ 


\section{CY 2010 SAP BOTTLE LISTS}

\section{Anions, MET-PMS}

\begin{tabular}{||l|c|c|c||}
\hline \multicolumn{1}{|c|}{ Parameter } & Lab Tests & $\begin{array}{c}\text { Chemical } \\
\text { Preservative }\end{array}$ & Bottle Types/Size \\
\hline \hline Anions & ANIONS & None & $1-250 \mathrm{~mL}$ polyethylene \\
\hline \hline Nitrate & $\mathrm{NO}-\mathrm{N}$ & $\begin{array}{c}\mathrm{H}_{2} \mathrm{SO}_{4} \text { to } \mathrm{pH}< \\
2 ; 4^{\circ}+/-2^{\mathrm{O}}\end{array}$ & $1-100 \mathrm{~mL}$ polyethylene \\
\hline \hline MET-PMS (ICP-MS) & ICPMS6020-EXT & $\mathrm{HNO3}$ & $1-250 \mathrm{~mL}$ polyethylene \\
\hline
\end{tabular}

Parameter:

Anions

$\operatorname{VOC}(1)$

$\operatorname{MET}(1)$

\section{LIMS LAB TEST ID}

ANIONS

VOA8260GW

ICPMS6020-EXT

${ }^{1}$ All samples chilled to $4^{\circ} \mathrm{C}+/-2^{\circ} \mathrm{C}$ 


\section{CY 2010 SAP BOTTLE LISTS}

\section{MET-PMS, RAD $(1,3)$}

\begin{tabular}{||c|c|c|c||}
\hline Parameter & Lab Tests & $\begin{array}{c}\text { Chemical } \\
\text { Preservative }\end{array}$ & Bottle Types/Size \\
\hline \hline MET-PMS (ICP-MS) & ICPMS6020-EXT & $\mathrm{HNO}_{3}$ & $1-250 \mathrm{~mL}$ polyethylene \\
\hline Radiochemistry (UV) & $\begin{array}{c}\text { GROSSAB-ENV } \\
\text { ASPECU-ENV }\end{array}$ & $\mathrm{HNO}_{3}$ & $1-1$ L polyethylene \\
\hline
\end{tabular}

Parameters: LIMS LAB TEST ID

MET(1) ICPMS6020-EXT

RAD(1)GROSSAB-ENV

RAD(3)ASPECU-ENV

${ }^{1}$ All samples chilled to $4^{\circ} \mathrm{C}+/-2^{\circ} \mathrm{C}$ 


\section{CY 2010 SAP BOTTLE LISTS}

\section{MET-PMS, VOC(1)}

\begin{tabular}{||l|c|c|c||}
\hline \multicolumn{1}{|c|}{ Parameter } & Lab Tests & $\begin{array}{c}\text { Chemical } \\
\text { Preservative }\end{array}$ & Bottle Types/Size \\
\hline \hline MET-PMS (ICP-MS) & ICPMS6020-EXT & $\mathrm{HNO}_{3}$ & $1-250 \mathrm{~mL}$ polyethylene \\
\hline Volatiles & VOA8260GW & None & $\begin{array}{l}2-40 \mathrm{~mL} \text { amber glass } \\
\text { with Teflon lined septum } \\
\text { lids }\end{array}$ \\
\hline
\end{tabular}

Parameters: LIMS LAB TEST ID

MET(1) ICPMS6020-EXT

VOC(1) VOA8260GW

${ }^{1}$ All samples chilled to $4{ }^{\circ} \mathrm{C}+/-2^{\circ} \mathrm{C}$ 


\section{CY 2010 SAP BOTTLE LISTS}

\section{Anions, MET-PMS, VOC (1)}

\begin{tabular}{||l|c|c|l||}
\hline \multicolumn{1}{|c|}{ Parameter } & Lab Tests & $\begin{array}{c}\text { Chemical } \\
\text { Preservative }\end{array}$ & \multicolumn{1}{|c||}{ Bottle Types/Size } \\
\hline \hline Anions & ANIONS & None & $1-250 \mathrm{~mL}$ polyethylene \\
\hline \hline Nitrate & NO3-N & $\begin{array}{c}\mathrm{H}_{2} \mathrm{SO}_{4} \text { to } \mathrm{pH}< \\
2 ; 4^{\mathrm{O}}+/-2^{\mathrm{O}}\end{array}$ & $1-100 \mathrm{~mL}$ polyethylene \\
\hline \hline MET-PMS (ICP-MS) & ICPMS6020-EXT & $\mathrm{HNO3}$ & $1-250 \mathrm{~mL}$ polyethylene \\
\hline \hline Volatiles & VOA8260GW & None & $\begin{array}{l}2-40 \mathrm{~mL} \text { amber glass } \\
\text { with Teflon lined } \\
\text { septum lids }\end{array}$ \\
\hline
\end{tabular}

Parameter:

Anions

$\operatorname{VOC}(1)$

$\operatorname{MET}(1)$

$\operatorname{VOC}(1)$

\section{LIMS LAB TEST ID}

ANIONS

VOA8260GW

ICPMS6020-EXT

VOA8260GW

${ }^{1}$ All samples chilled to $4{ }^{\circ} \mathrm{C}+/-2^{\circ} \mathrm{C}$ 


\section{ESTABLISHED HOLDING TIMES}

\begin{tabular}{|l|l|}
\hline Parameter & Holding Times \\
\hline Alkalinity (Carbonate, Bicarbonate) & 14 days \\
\hline Anions (Chloride, Nitrate, and Sulfate) & 28 days \\
\hline Fluoride & 28 days \\
\hline Mercury & 28 days \\
\hline Metals (ICP, ICPMS) & 6 months \\
\hline Radiochemistry & 6 months \\
\hline Solids, Total Dissolved & 7 days \\
\hline Solids, Total Suspended & 7 days \\
\hline VOA & 7 days \\
\hline
\end{tabular}

\section{ESTABLISHED TURNAROUND TIMES}

The Groundwater Protection Program and the Analytical Chemistry Organization (ACO) laboratory have agreed upon a turnaround time, such that the analytical data generated from each sampling location will be completed within 35 days of receipt. Every two weeks, data that has been approved since the previous twoweek period will be transmitted in the form of hard copy of the approved lab reports for each location, along with an electronic copy in a standardized and compatible format (please see the most recent version of the Y-12 Plant Groundwater Protection Program Data Management Plan).

\section{ELEVATED MINIMUM DETECTABLE ACTIVITY}

Groundwater samples with high TDS (>1,000 mg/L) typically have elevated minimum detectable activities (MDAs) for gross alpha (>15 pCi/L) and gross beta (> $50 \mathrm{pCi} / \mathrm{L})$. However, the MDAs for specific isotopic analyses are unaffected by the sample solid content. For samples with gross activity results that are less than an elevated MDA, and specific isotopic analyses have not been requested, the laboratory will issue a request to analyze for the principal alpha- or beta-emitting isotopes. That is, if the gross alpha MDA exceeds $15 \mathrm{pCi} / \mathrm{L}$ and the result is less than $15 \mathrm{pCi} / \mathrm{L}$, then the laboratory will request analyses of isotopic uranium (by method Y/P65-7061). Similarly, if a sample has an elevated gross beta MDA ( $>50 \mathrm{pCi} / \mathrm{L})$ and the result is less than the MDA, then the laboratory would request analysis of technetium-99 activity. These requests will be approved by the Y-12 Groundwater Protection Program manager, or designee, before analyses are performed. 
APPENDIX F

MANAGEMENT OF PURGED GROUNDWATER 
APPENDIX F.1

WASTE MANAGEMENT PLAN 


\section{Approvals}

Don Bohrman [signature on file]

Don Bohrman

$12 / 18 / 06$

Date

BWXT Y-12, LLC

Environmental Officer

Mary Wiginton [signature on file]

Mary Wiginton

$12 / 18 / 06$

BWXT Y-12, LLC

Waste Engineer

Date

Tom Conrad for D. McCune [signature on file]

12/18/06

Dave McCune

Date

Bechtel Jacobs, LLC

Waste Treatment Operations

Mark Burris [signature on file]

$12 / 18 / 06$

Brad E Skaggs or Mark S. Burris

Date

BWXT Y-12, LLC

Environmental Compliance 
Waste Management Plan for Y-12 Groundwater Protection Program Sampling Activities

\begin{tabular}{|l|l|l|l|l|}
\hline \multicolumn{1}{|c|}{ Waste stream } & \multicolumn{1}{|c|}{ Characterization ${ }^{3}$} & Segregation Requirements & \multicolumn{1}{c|}{ Packaging } & \multicolumn{1}{c|}{ Disposal Path } \\
\hline $\begin{array}{l}\text { Purge water }{ }^{2} \text { that is not } \\
\text { contained }\end{array}$ & $\begin{array}{l}\text { Non-hazardous, non-radiological } \\
\text { contaminated waters. Analytical results } \\
\text { indicate constituents in the water are less } \\
\text { than Safe Drinking Water Act Maximum } \\
\text { Contaminant Levels (MCL). In } \\
\text { addition, historical knowledge of } \\
\text { relevant groundwater plumes at the Y-12 } \\
\text { National Security Complex confirm the } \\
\text { non-detection of contaminants, or the } \\
\text { detection of contaminants (J values), but } \\
\text { still below the MCL. See the most } \\
\text { current GWPP Groundwater Monitoring } \\
\text { Data Compendium }\end{array}$ & Not contained & $\begin{array}{l}\text { ACO technicians will } \\
\text { dispense/dispose of waters directly } \\
\text { to ground surface at the well } \\
\text { location. }\end{array}$ & \\
\hline
\end{tabular}




\begin{tabular}{|c|c|c|c|c|}
\hline Waste stream & Characterization $^{3}$ & Segregation Requirements & Packaging & Disposal Path \\
\hline $\begin{array}{l}\text { SID }^{1} 2212 \text { purge water } \\
\text { (non-regulated and non- } \\
\text { hazardous purge water) }\end{array}$ & $\begin{array}{l}\text { Non-hazardous, contaminated waste } \\
\text { waters. Analytical results indicate } \\
\text { concentration in the water exceed the } \\
\text { MCL. These waters can contain nitrate } \\
\text { concentration }>100 \mathrm{mg} / \mathrm{L} \text {, Uranium } \\
>0.03 \mathrm{mg} / \mathrm{L} \text {, and Uranium isotopes }>4 \% \\
\text { of DCG. Waters commonly contain the } \\
\text { following typical halogenated } \\
\text { compounds (not inclusive) that exceed } \\
\text { the MCL, but are below RCRA TCLP } \\
\text { levels, include: Tetrachloroethene, } \\
\text { Trichloroethene, cis-1,2-Dichloroethene, } \\
\text { Carbon Tetrachloride, } 1,1- \\
\text { Dichloroethane, Methylene Chloride, } \\
\text { and Vinyl Chloride. Other } \\
\text { radioisotopes present consist of Tc-99 } \\
\text { and daughter products of Uranium. } \\
\text { Although not regulated, this waste water } \\
\text { is contained, handled, and sent for } \\
\text { disposal as a Best Management Practice } \\
\text { (BMP) at Y-12. As a BMP, this } \\
\text { contaminated purge water is not place on } \\
\text { clean surfaces (soils) or near surface } \\
\text { water tributaries. Annual groundwater } \\
\text { data evaluation, multiple sampling } \\
\text { event, and groundwater plume } \\
\text { characteristics provide ample evidence } \\
\text { of this classification. }\end{array}$ & $\begin{array}{l}\text { Segregate non-regulated waste } \\
\text { waters from other GWPP } \\
\text { waste waters that contain a } \\
\text { RCRA hazardous waste (SID } \\
2214 \text { and 2216). } \\
\text { Waste streams }{ }^{1} \text { have been } \\
\text { characterized and established } \\
\text { per well location and are } \\
\text { published in GWPP's annual } \\
\text { GWPP Sampling and Analysis } \\
\text { Plan. } \\
\text { Waters can be combined and } \\
\text { bulk as necessary in a DOT } \\
\text { approved container. }\end{array}$ & $\begin{array}{l}\text { Place in a DOT approved } \\
\text { container. } \\
\text { The above containers are } \\
\text { compatible with the } \\
\text { purge water and meet } \\
\text { packaging requirements } \\
\text { specified in Master } \\
\text { Profile WW-01 } \\
\text { Label containers in } \\
\text { accordance with Y71- } \\
\text { 310, Waste Container } \\
\text { Labeling }\end{array}$ & $\begin{array}{l}\text { SID } 2212 \text { waste stream meets the } \\
\text { waste acceptance criteria of Master } \\
\text { Profile WW-01. Sampling data is } \\
\text { used to complete Attachment G of } \\
\text { UCN 2109. If Uranium is present, } \\
\text { above detection levels, then a wt } \\
\% \text { OU5 sample is required to } \\
\text { determine enrichment, and a } \\
\text { duplicate sample is required if } \\
\text { results are >0.93 wt U235. All } \\
\text { other constituents listed in WW-01 } \\
\text { have been quantified through } \\
\text { current analytical results, previous } \\
\text { analyses, historical data (prior to } \\
\text { 1996), and groundwater plume } \\
\text { composition. A Process Knowledge } \\
\text { form attached to each UCN } 2109 \\
\text { documents the presence of } \\
\text { constituents seen in SID } 2212 \\
\text { waters and the absence of other such } \\
\text { constituents. This waste stream is } \\
\text { disposed at Y-12 National Security } \\
\text { Complex's onsite treatment facility } \\
\text { with authorization from Waste } \\
\text { Treatment Operations. Depending } \\
\text { on enrichment content, normal } \\
\text { disposal would be at either the West } \\
\text { End Treatment Facility (WETF) or } \\
\text { the Central Pollution Control } \\
\text { Facility (CPCF). }\end{array}$ \\
\hline
\end{tabular}




\begin{tabular}{|c|c|c|c|c|}
\hline Waste stream & Characterization $^{3}$ & Segregation Requirements & Packaging & Disposal Path \\
\hline $\begin{array}{l}\text { SID }^{1} 2214 \text { purge water } \\
\text { (purge water from } \\
\text { multiple F-listed RCRA } \\
\text { groundwater wells, along } \\
\text { with rinse waters from } \\
\text { sampling equipment and } \\
\text { disposables, bulked into } \\
\text { the same drum. All waste } \\
\text { water carries the F039 } \\
\text { waste code). }\end{array}$ & $\begin{array}{l}\text { Hazardous waste waters (no radiological } \\
\text { contaminants). Characterization based } \\
\text { upon well location. Wells located down- } \\
\text { gradient of the Bear Creek Burial } \\
\text { Grounds between north tributary (NT) } 6 \\
\text { and NT 8, and north of Bear Creek. } \\
\text { Purge water most likely contains } \\
\text { leachate from the BCBGs and is } \\
\text { considered RCRA F-listed (40 CFR Part } \\
\text { 261.31) based on established } \\
\text { documentation (F039 leachate is } \\
\text { comprised of F codes: F001, F002, } \\
\text { F004, and F005). } \\
\text { Typical halogenated volatile organic } \\
\text { compounds detected in the SID 2214 } \\
\text { waters, which are above the MCL } \\
\text { include: Tetrachloroethene, } \\
\text { Trichloroethene, 1,2-Dichloroethene, } \\
\text { 1,1-Dichloroethene, } \\
\text { 1,1,1-Trichloroethane, 1,1- } \\
\text { Dichloroethane, Methylene Chloride, } \\
\text { and Vinyl Chloride. Typically Benzene } \\
\text { and other total petroleum hydrocarbons } \\
\text { have also been identified. }\end{array}$ & $\begin{array}{l}\text { Segregate RCRA F-listed } \\
\text { waste waters from non- } \\
\text { regulated waste waters (SID } \\
\text { 2212) and RCRA } \\
\text { Characteristic waste waters } \\
\text { (SID 2216). } \\
\text { Waste waters are } \\
\text { bulked/accumulated at RCRA } \\
\text { Satellite Accumulation Area } \\
\text { (SAA) \#SA-993, under the } \\
\text { direction of the SAA Operator } \\
\text { or Alternate Operator }\end{array}$ & $\begin{array}{l}\text { Place in a DOT approved } \\
\text { container. } \\
\text { Waste is transported as } \\
\text { DOT Class } 9 \text { under a } \\
\text { Bill of Lading listing the } \\
\text { assigned EPA waste } \\
\text { code. Transporter has } \\
\text { received DOT training. } \\
\text { The above containers are } \\
\text { compatible with the } \\
\text { purge water and meet } \\
\text { packaging requirements } \\
\text { specified in Master } \\
\text { Profile WW-01 } \\
\text { Label containers in } \\
\text { accordance with Y71- } \\
\text { 310, Waste Container } \\
\text { Labeling }\end{array}$ & $\begin{array}{l}\text { Send SID } 2214 \text { waste waters to 90- } \\
\text { Day Yard for further management. } \\
\text { RCRA F-listed waste are } \\
\text { prohibited under Master Profile } \\
\text { WW-01, except under special } \\
\text { arrangement with DOE, or approved } \\
\text { by Waste Treatment Coordinator for } \\
\text { waste that can be treated at CPCF or } \\
\text { Groundwater Treatment Facility } \\
\text { (GWTF). SID } 2214 \text { waste waters } \\
\text { have been approved for treatment at } \\
\text { GWTF with the following } \\
\text { prohibitions: waters with Uranium } \\
\text { above detection (based on waste } \\
\text { sample analyses) and Nitrates in } \\
\text { concentration > 100 mg/L. All } \\
\text { other constituents listed in WW-01 } \\
\text { have been quantified through } \\
\text { current analytical results, previous } \\
\text { analyses, historical data (prior to } \\
\text { 1996), and groundwater plume } \\
\text { composition. A Process Knowledge } \\
\text { form attached to each UCN } 2109 \\
\text { documents the presence and absence } \\
\text { of WW-01 constituents seen in SID } \\
\text { 2214 waters. This waste stream is } \\
\text { disposed at Y-12 National Security } \\
\text { Complex's onsite treatment facility } \\
\text { with authorization from Waste } \\
\text { Treatment Operations. }\end{array}$ \\
\hline
\end{tabular}




\begin{tabular}{|c|c|c|c|c|}
\hline Waste stream & Characterization $^{3}$ & Segregation Requirements & Packaging & Disposal Path \\
\hline $\begin{array}{l}\text { SID }^{1} 2216 \text { purge water } \\
\text { (purge water from } \\
\text { multiple RCRA } \\
\text { characteristic wells } \\
\text { bulked into the same } \\
\text { drum. The EPA waste } \\
\text { code is dependent on the } \\
\text { well location). }\end{array}$ & $\begin{array}{l}\text { Hazardous waste waters (mixed and } \\
\text { non-radiological contaminated). } \\
\text { Analytical results indicate that } \\
\text { concentrations exceed a RCRA Toxicity } \\
\text { Contaminant Leaching Procedure (TCLP } \\
\text { - 40 CFR Part 261.24). Annual } \\
\text { groundwater data evaluation, plume } \\
\text { evaluations, and repeated sampling } \\
\text { events give weighted evidence to this } \\
\text { classification (wells may receive this } \\
\text { classification if concentrations have } \\
\text { been consistently approaching the } \\
\text { RCRA TCLP levels). } \\
\text { SID 2216 waste water can contain the } \\
\text { following EPA waste codes: } \\
\text { D005 - Barium } \\
\text { D006 - Cadmium } \\
\text { D018 - Benzene } \\
\text { D019 - Carbon Tetrachloride } \\
\text { D029 - 1,1-Dichloroethene } \\
\text { D039 - Tetrachloroethene } \\
\text { D040 - Trichloroethene } \\
\text { D043 - Vinyl Chloride } \\
\text { In addition to the above, these waters } \\
\text { may contain the following volatile } \\
\text { organic compounds: } 1,2-D i c h l o r o e t h e n e, \\
1,1,2-\text { Trichloro-1,2,2-triflouroethane, } \\
\text { 1,1,1-Trichloroethane, } \\
\text { Dichloroethane, Acetone, Methylene } \\
\text { Chloride, Chloroform and other total } \\
\text { petroleum hydrocarbons. These waters } \\
\text { may can contain trace metals, nitrate } \\
\text { concentration > 100 mg/L, Uranium } \\
>0.03 \text { mg/L, Uranium isotopes }>4 \% \text { of } \\
\text { DCG, and other radioisotopes (Tc99 and } \\
\text { daughter products of Uranium). }\end{array}$ & $\begin{array}{l}\text { Segregate RCRA } \\
\text { characteristic waste waters } \\
\text { from non-regulated waste } \\
\text { waters (SID 2212) and RCRA } \\
\text { F-listed waste waters (SID } \\
\text { 2216). } \\
\text { RCRA characteristic waste } \\
\text { waters are bulked/accumulated } \\
\text { at RCRA Satellite } \\
\text { Accumulation Area (SAA) } \\
\text { \#SA-992, under the direction } \\
\text { of the SAA Operator or } \\
\text { Alternate Operator }\end{array}$ & $\begin{array}{l}\text { Place in a DOT approved } \\
\text { container. } \\
\text { Waste is transported as } \\
\text { DOT Class } 9 \text { under a } \\
\text { Bill of Lading listing the } \\
\text { assigned EPA waste } \\
\text { code. Transporter has } \\
\text { received DOT training. } \\
\text { The above containers are } \\
\text { compatible with the } \\
\text { purge water and meet } \\
\text { packaging requirements } \\
\text { specified in Master } \\
\text { Profile WW-01 } \\
\text { Label containers in } \\
\text { accordance with Y71- } \\
\text { 310, Waste Container } \\
\text { Labeling }\end{array}$ & $\begin{array}{l}\text { Send SID } 2216 \text { waste waters to } 90- \\
\text { Day Yard for further management. } \\
\text { SID } 2216 \text { waste waters meet the } \\
\text { waste acceptance criteria of Master } \\
\text { Profile WW-01. Waters with } \\
\text { Uranium above detection (based on } \\
\text { waste sample analyses) require a wt } \\
\% \text { 235 sample to determine } \\
\text { enrichment and a duplicate sample } \\
\text { is required if results are }>0.93 \text { wt } \\
\text { U235. Nitrates concentration }>10 \\
\text { mg/L must be indicated. All other } \\
\text { constituents listed in WW-01 have } \\
\text { been quantified through current } \\
\text { analytical results, previous analyses, } \\
\text { historical data (prior to 1996), and } \\
\text { groundwater plume composition. A } \\
\text { Process Knowledge form attached to } \\
\text { each UCN } 2109 \text { documents the } \\
\text { presence and absence of WW-01 } \\
\text { constituents seen in SID } 2216 \\
\text { waters. This waste stream is } \\
\text { disposed at Y-12 National Security } \\
\text { Complex's onsite treatment facility } \\
\text { with authorization from Waste } \\
\text { Treatment Operations. Depending } \\
\text { on enrichment content, waste waters } \\
\text { are disposed at the West End } \\
\text { Treatment Facility (WETF) or the } \\
\text { Central Pollution Control Facility } \\
\text { (CPCF). }\end{array}$ \\
\hline
\end{tabular}




\begin{tabular}{|c|c|c|c|c|}
\hline Waste stream & Characterization $^{3}$ & Segregation Requirements & Packaging & Disposal Path \\
\hline $\begin{array}{l}\text { Disposables and sampling } \\
\text { equipment in contact } \\
\text { with RCRA } \\
\text { characteristic or F-listed } \\
\text { purge water: } \\
\text { Sampling equipment: } \\
\text { includes sample pumps, } \\
\text { tubing, sample trays, and } \\
\text { flow-through cells (all } \\
\text { components). These items } \\
\text { meet the definition of a } \\
\text { "container” under RCRA. } \\
\text { Non-absorbent } \\
\text { disposables - include: } \\
\text { gloves, plastic bags, and } \\
\text { instrument probes } \\
\text { Absorbent disposables - } \\
\text { includes paper towels, } \\
\text { wipes, clothes, litmus } \\
\text { paper. During normal } \\
\text { sampling operations these } \\
\text { items should not come } \\
\text { into contact with RCRA } \\
\text { characteristic or F-listed } \\
\text { waste waters. }\end{array}$ & $\begin{array}{l}\text { Non-hazardous solid waste and RCRA } \\
\text { empty containers. The sampling } \\
\text { equipment and disposables which comes } \\
\text { in contact with RCRA purge waters will } \\
\text { not be subject to RCRA if: } \\
\text { 1) The waste can be sufficiently } \\
\text { removed from non-absorbent material } \\
\text { (disposables), such as nitrile gloves, } \\
\text { plastic surfaces, instrument probes, and } \\
\text { external surfaces of sample bottles by } \\
\text { rinsing such items. All rinse water must } \\
\text { be collected and bulked under the } \\
\text { appropriate RCRA waste stream (SID } \\
\text { 2216 or } 2214 \text { ). } \\
\text { 2) Sampling equipment that meets the } \\
\text { definition of a "container" under RCRA } \\
\text { and is not subject to regulation once } \\
\text { the container is “empty" as defined } \\
\text { under } 40 \text { CFR Part 261.7, paragraph } \\
\text { (b). To meet this requirement all fluids } \\
\text { must be sufficiently drained from the } \\
\text { equipment, by normal means as possible, } \\
\text { and then rinsed at least once to remove } \\
\text { residue. All rinse water must be } \\
\text { collected and bulked under the } \\
\text { appropriate RCRA waste stream (SID } \\
2216 \text { or } 2214 \text { ). } \\
\text { 3) Absorbent disposable such as wipes, } \\
\text { paper towels, or clothes that are use to } \\
\text { remove/clean/dry any addition } \\
\text { liquids/residues RCRA empty } \\
\text { containers, once the items are rinsed, are } \\
\text { also not subject to RCRA. Litmus } \\
\text { paper, if used for its intended purpose, } \\
\text { and does not come into contact with } \\
\text { F039 waste water, is also not subject to } \\
\text { RCRA. }\end{array}$ & $\begin{array}{l}\text { Segregate non-regulated } \\
\text { waste streams from those } \\
\text { items subject to RCRA. all } \\
\text { Sampling equipment: can be } \\
\text { reused as necessary for the } \\
\text { multiple sampling events and } \\
\text { are not regulated. } \\
\text { Non-absorbent disposables - } \\
\text { once rinsed are not regulated. } \\
\text { Absorbent disposables -. } \\
\text { Items used to wipe/dry/clean } \\
\text { RCRA empty containers are } \\
\text { not regulated and can also be } \\
\text { disposed of into sanitary trash } \\
\text { (profile S-020). If these items } \\
\text { do come into contact with } \\
\text { RCRA waste water, the items } \\
\text { are subject to regulation. } \\
\text { These items must be wrung } \\
\text { out as much as possible (water } \\
\text { collected) and segregated from } \\
\text { non-regulated items. }\end{array}$ & $\begin{array}{l}\text { ALL non-regulated } \\
\text { items - dispose of into } \\
\text { the appropriate sanitary } \\
\text { waste receptacle or } \\
\text { dumpster, as specified } \\
\text { under Master Profile S- } \\
020 \text {. } \\
\text { Any items subject to } \\
\text { RCRA regulation must } \\
\text { be place in a DOT } \\
\text { approved container and } \\
\text { labeled in accordance } \\
\text { with procedure Y71-310, } \\
\text { Waste Container } \\
\text { Labeling. Waste is } \\
\text { transported as DOT } \\
\text { Class } 9 \text { under a Bill of } \\
\text { Lading, listing the } \\
\text { assigned EPA waste } \\
\text { code, to the 90-Day Yard } \\
\text { for further management. } \\
\text { Transporter has received } \\
\text { DOT training. }\end{array}$ & $\begin{array}{l}\text { All the items listed below require } \\
\text { authorization from Y-12 Waste } \\
\text { Management prior to disposal in } \\
\text { Sanitary Trash } \\
\text { Sampling equipment - once the } \\
\text { item is no longer of use, or can no } \\
\text { longer be used, the item can be } \\
\text { disposed of in sanitary trash (Waste } \\
\text { Profile No. S-020). } \\
\text { Non-absorbent disposables - after } \\
\text { items are rinsed, collect the rinse } \\
\text { solution and bulk with SID } 2216 \text { or } \\
\text { 2214 purge water, and then dispose } \\
\text { of the item in sanitary trash (S-020). } \\
\text { Absorbent disposables - not } \\
\text { subject to regulation can be } \\
\text { disposed into sanitary trash (S-020). } \\
\text { Absorbent material that comes } \\
\text { into contact with } \\
\text { Characteristic (SID 2216) purge } \\
\text { water, by process knowledge the } \\
\text { whole material if tested under the } \\
\text { TCLP would not exceed TCLP } \\
\text { levels, and therefore the item can be } \\
\text { disposed into sanitary trash (S-020). } \\
\text { Absorbent material that comes } \\
\text { into contact with RCRA F-listed } \\
\text { waste waters (SID 2214) will be } \\
\text { subject to regulation and must be } \\
\text { send to the } 90-D a y \text { Yard for further } \\
\text { management (Master Profile HW- } \\
\text { 01). Final disposal path will be } \\
\text { determined by Navarro-GEM to an } \\
\text { off-site RCRA TSD. }\end{array}$ \\
\hline
\end{tabular}




\section{Waste Management Plan for Y-12 Groundwater Protection Program Sampling Activities}

\begin{tabular}{|c|c|c|c|c|}
\hline Waste stream & Characterization $^{3}$ & Segregation Requirements & Packaging & Disposal Path \\
\hline $\begin{array}{l}\text { All disposables and } \\
\text { equipment used for } \\
\text { GWPP purposes (non F- } \\
\text { listed wells): } \\
\text { Sampling pumps, gloves, } \\
\text { wipes, tubing, litmus } \\
\text { paper, instrument probes, } \\
\text { sample trays, and flow- } \\
\text { through cells. }\end{array}$ & $\begin{array}{ll}\text { Non-hazardous solid } & \text { waste. } \\
\text { Characterization is not required. } & \end{array}$ & $\begin{array}{l}\text { Segregate F-listed } \\
\text { contaminated items from non } \\
\text { F-listed contaminated items. }\end{array}$ & N/A & $\begin{array}{l}\text { Items not in contact with any F- } \\
\text { listed purge water can be disposed } \\
\text { in sanitary trash (profile S020) with } \\
\text { authorization from Y-12 Waste } \\
\text { Management. } \\
\text { All sampling equipment is to be } \\
\text { reused till the item is no longer of } \\
\text { use and then disposed of in sanitary } \\
\text { trash. } \\
\text { All Sanitary waste placed in the } \\
\text { approved on-site Solid Waste } \\
\text { Disposal Facility (Industrial } \\
\text { Landfill) }\end{array}$ \\
\hline $\begin{array}{l}\text { Waters/Fluids generated } \\
\text { during well development } \\
\text { of existing wells (well } \\
\text { development is } \\
\text { performed on an as } \\
\text { needed basis, prior to } \\
\text { sampling, to maintain } \\
\text { groundwater flow to well. } \\
\text { Five to } 10 \text { well casing } \\
\text { volumes are generated) }\end{array}$ & $\begin{array}{l}\text { Well development of existing wells will } \\
\text { utilize the most recent sampling } \\
\text { analytical results and will follow the } \\
\text { three waste streams (SIDs) for purge } \\
\text { water. }\end{array}$ & $\begin{array}{l}\text { Segregate water based on the } \\
\text { three existing waste streams } \\
\text { for purge water }\end{array}$ & $\begin{array}{l}\text { Based on volume and } \\
\text { waste stream ID number. } \\
\text { Containers could consist } \\
\text { of drums, polytanks, or } \\
\text { tankers. }\end{array}$ & $\begin{array}{l}\text { See the three purge water waste } \\
\text { streams IDs above }\end{array}$ \\
\hline
\end{tabular}

1 “SID” - “Stream Identification Number” are the pre-established waste streams identification (ID) for purge waters generated at Y-12. These waste streams were established by Y-12 Waste Operations, prior to 1995, and have been utilized to segregate waste waters. The waste stream ID is established for the coming Calendar Year (CY) for each well location to be monitored; based upon characterization of the most recent sampling results for that well location. These are published an appendix to GWPP's annual Sampling and Analysis Plan (published 2-3 months prior the start of the CY), and the waste stream is established for any other wells added during that CY and documented in addenda to this plan.

"Purge Water" - unusable portion of groundwater purged from a well prior to sample collection. Water is in a liquid form,(99.9\% liquid) with normally < 100 mg/L of suspended solids. Water contains contaminants that are in solution (dissolved phase) with little sediment load.

${ }^{3}$ Analytical results (past and present) from sampling events are used to characterize purge water. The GWPP uses a standardized parameter list for every sample, which includes:

1. ICP metals (SW846- EPA 6010B), ICPMS metals (EPA-200.8), Mercury (SW846 - 7470) - includes Uranium metal (0.0005 mg/L)

2. Anions - Alkalinity, Chloride, Fluoride, Nitrates, Sulfates

3. Volatile Organic Compound - SW846 - EPA 8260B

4. Gross Alpha and Gross Beta (EPA-900.0)

The following radioisotopes have been analyzed for on an as needed basis: Tritium, Tc-99, Isotopic Uranium, Total Uranium and wt\% U235, and other heavy radioisotopes (Am241, Np 237, I129, Thorium, Radium). 


\section{Appendix F.1}

\section{Y-12 GWPP PURGE WATER MANAGEMENT}

Example of Waste Identification Tag (UCN 2114B) for SID 2212 purge water

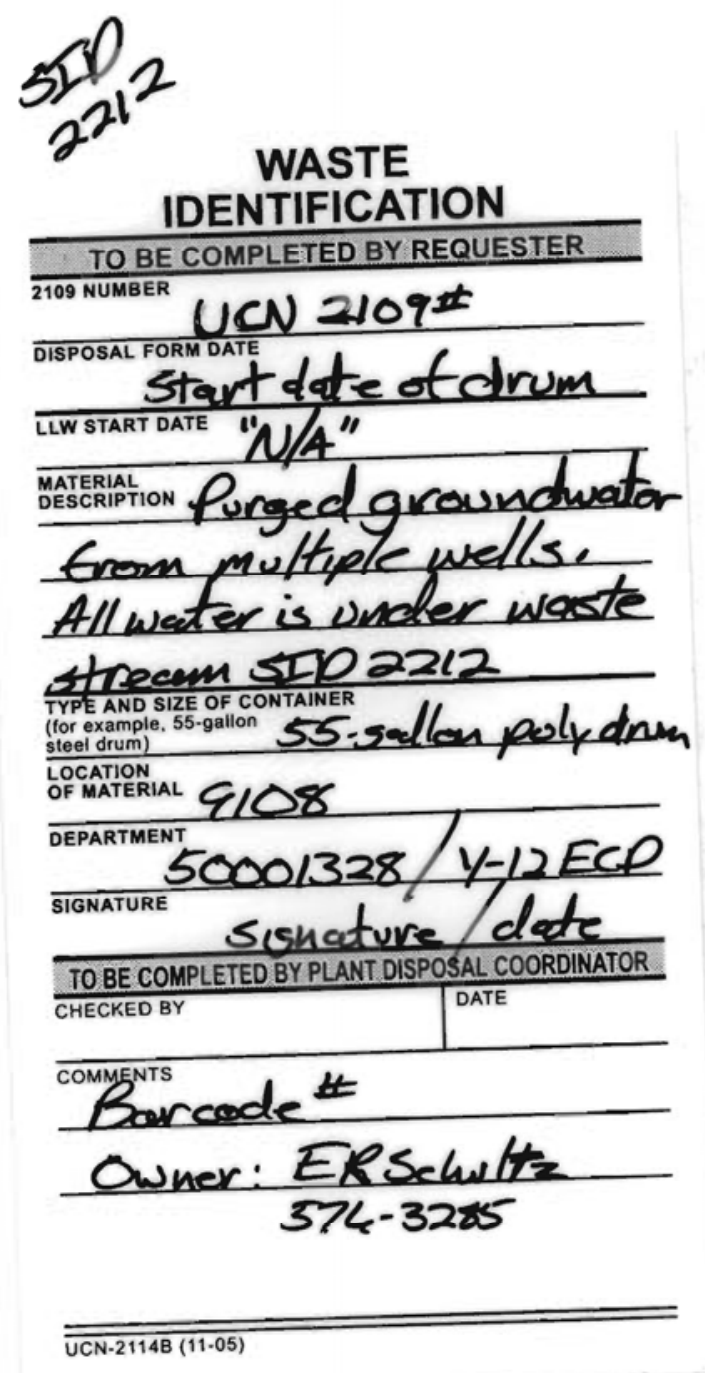




\section{Appendix F.1}

\section{Y-12 GWPP PURGE WATER MANAGEMENT}

Example of Hazardous Waste Identification Tag (UCN 2114A) for SID 2214 purge water

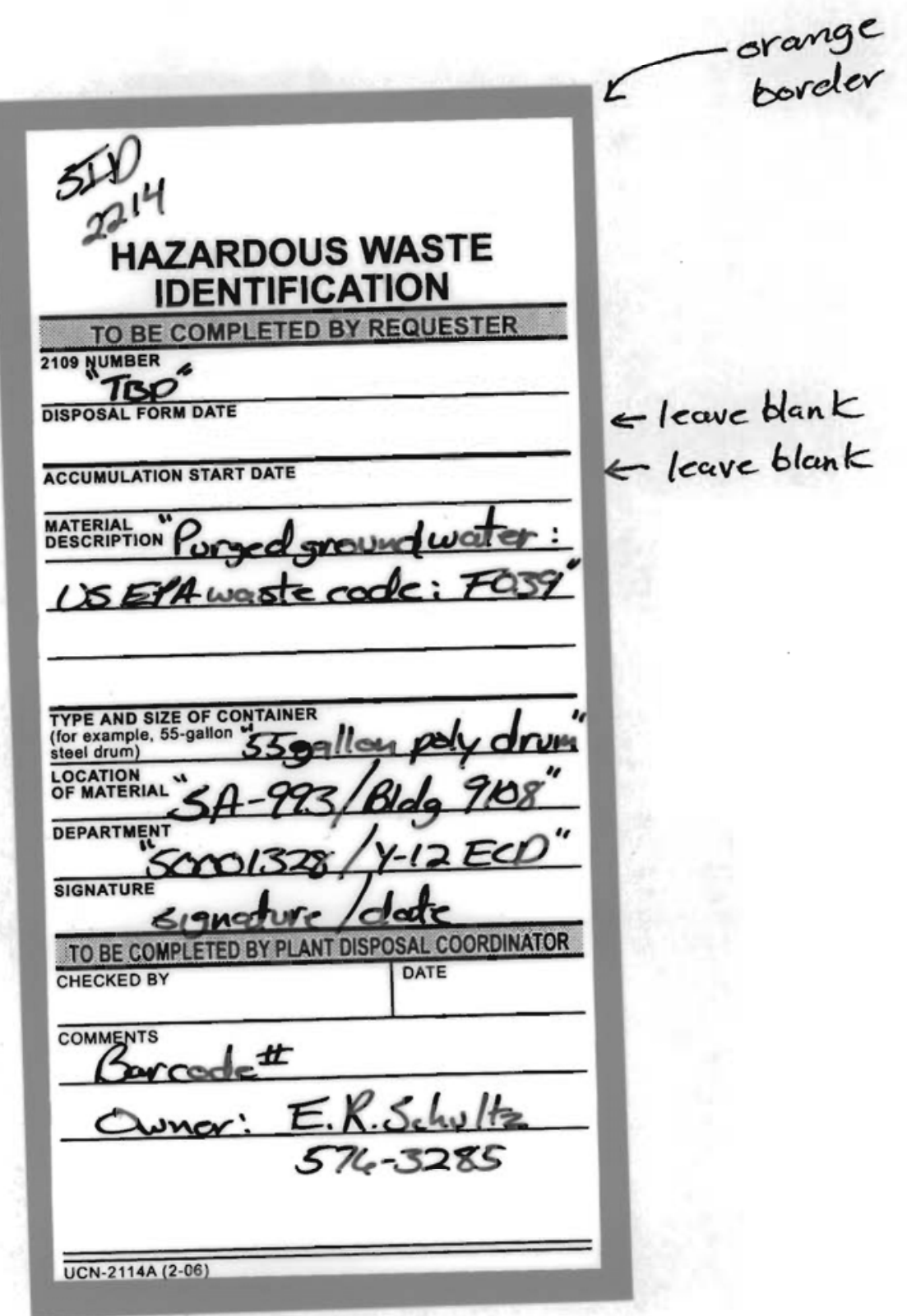




\section{Appendix F.1}

\section{Y-12 GWPP PURGE WATER MANAGEMENT}

Example of Hazardous Waste Identification Tag (UCN 2114A) for SID 2216 purge water
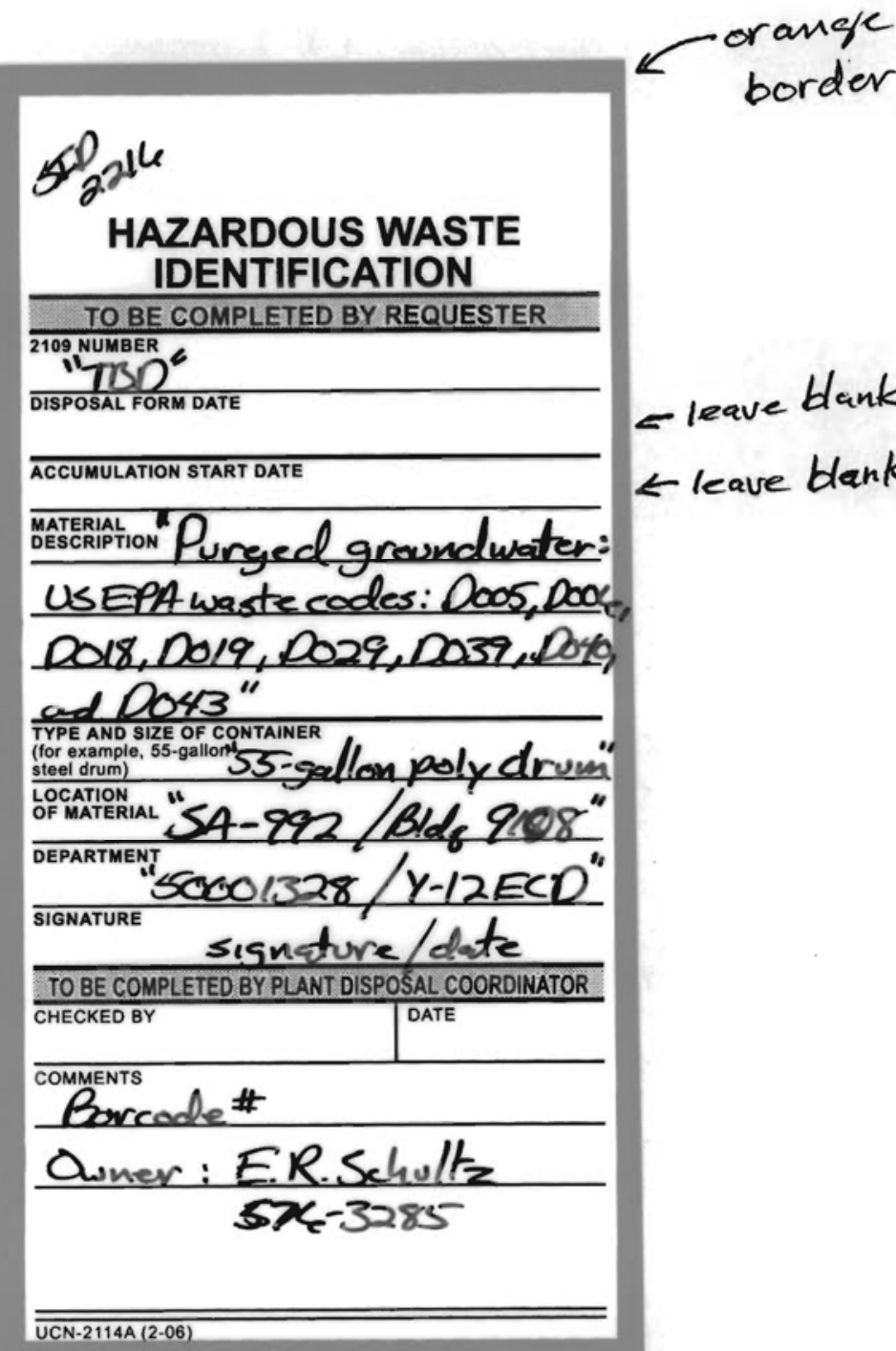

- leave blank $\leftarrow$ leave blank 
APPENDIX F.2

WASTEWATER STREAM IDENTIFICATION FOR PURGED GROUNDWATER 
Table F.2. Waste stream identification (SID) and RCRA waste code for groundwater purged from wells to be sampled during CY 2010

\begin{tabular}{|c|c|c|c|c|c|c|c|}
\hline \multirow{2}{*}{ Regime } & \multirow{2}{*}{ Location } & \multicolumn{4}{|c|}{ CY 2010 Sampling Qtr. } & \multirow{2}{*}{$\begin{array}{c}\text { Waste Stream ID (SID } \\
\#)\end{array}$} & \multirow{2}{*}{ RCRA Waste Code } \\
\hline & & Q1 & Q2 & Q3 & Q4 & & \\
\hline \multirow[t]{36}{*}{ Bear Creek } & GW-006 & $\mathrm{Y}$ & & & & SID 2212 & \\
\hline & GW-014 & & & $\mathrm{Y}$ & & SID 2214 & F039 \\
\hline & GW-065 & $\mathrm{Y}$ & & $\mathrm{Y}$ & & SID 2212 & \\
\hline & GW-068 & & & $\mathrm{Y}$ & & SID 2214 & F039 \\
\hline & GW-071 & $\mathrm{Y}$ & & $\mathrm{Y}$ & & SID 2214 & F039 \\
\hline & GW-082 & $\mathrm{Y}$ & & & & SID 2214 & F039 \\
\hline & GW-085 & & & $\mathrm{Y}$ & & SID 2212 &. \\
\hline & GW-098 & & & $\mathrm{Y}$ & & SID 2212 & . \\
\hline & GW-100 & & & $\mathrm{Y}$ & & SID 2212 & \\
\hline & GW-101 & & & $\mathrm{Y}$ & & SID 2212 & . \\
\hline & GW-225 & & & $\mathrm{Y}$ & & SID 2212 & . \\
\hline & GW-229 & & & $\mathrm{Y}$ & & SID 2212 & 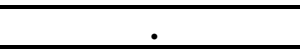 \\
\hline & GW-246 & $\mathrm{Y}$ & & & & SID 2212 & \\
\hline & GW-289 & $\mathrm{Y}$ & & & & SID 2214 & F039 \\
\hline & GW-291 & & & $\mathrm{Y}$ & & SID 2214 & F039 \\
\hline & GW-307 & & & $\mathrm{Y}$ & & SID 2212 & . \\
\hline & GW-310 & & & $\mathrm{Y}$ & & SID 2212 & $\dot{\square}$ \\
\hline & GW-312 & & & $\mathrm{Y}$ & & SID 2212 & . \\
\hline & GW-315 & & & $\mathrm{Y}$ & & SID 2212 & . \\
\hline & GW-365 & & & $\mathrm{Y}$ & & SID 2212 &. \\
\hline & GW-526 & & & $\mathrm{Y}$ & & SID 2212 & 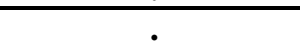 \\
\hline & GW-537 & $\mathrm{Y}$ & & & & SID 2212 & . \\
\hline & GW-601 & & & $\mathrm{Y}$ & & SID 2212 & \\
\hline & GW-615 & & & $\mathrm{Y}$ & & SID 2216 & D005 \\
\hline & GW-616 & $\mathrm{Y}$ & & & & SID 2212 & \\
\hline & GW-623 & & & $\mathrm{Y}$ & & SID 2214 & F039 \\
\hline & GW-626 & $\mathrm{Y}$ & & & & SID 2214 & F039 \\
\hline & GW-627 & $\mathrm{Y}$ & & $\mathrm{Y}$ & & SID 2214 & F039 \\
\hline & GW-648 & $\mathrm{Y}$ & & $\mathrm{Y}$ & & SID 2212 & \\
\hline & GW-653 & & & $\mathrm{Y}$ & & SID 2214 & F039 \\
\hline & GW-703 & & & $\mathrm{Y}$ & & SID 2212 &. \\
\hline & GW-724 & & & $\mathrm{Y}$ & & SID 2212 & . \\
\hline & GW-725 & & & $\mathrm{Y}$ & & SID 2212 & 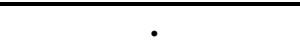 \\
\hline & GW-738 & & & $\mathrm{Y}$ & & SID 2212 & . \\
\hline & GW-740 & & & $\mathrm{Y}$ & & SID 2212 & . \\
\hline & GW-829 & $\mathrm{Y}$ & & & & SID 2212 & . \\
\hline \multirow[t]{4}{*}{ Chestnut Ridge } & GW-322 & & $\mathrm{Y}$ & & & SID 2212 & . \\
\hline & GW-514 & & $\mathrm{Y}$ & & & Not Contained & . \\
\hline & GW-608 & & $\mathrm{Y}$ & & & Not Contained & . \\
\hline & GW-609 & & $\mathrm{Y}$ & & & Not Contained & . \\
\hline \multirow[t]{10}{*}{ East Fork } & $55-2 B$ & & $\mathrm{Y}$ & & & SID 2212 & 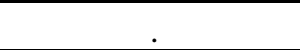 \\
\hline & $55-2 \mathrm{C}$ & & $\mathrm{Y}$ & & & SID 2212 & \\
\hline & $55-3 \mathrm{~A}$ & $\mathrm{Y}$ & & $\mathrm{Y}$ & & SID 2216 & D039, D040 \\
\hline & $55-3 \mathrm{~B}$ & $\mathrm{Y}$ & & $\mathrm{Y}$ & & SID 2216 & D039, D040, D043 \\
\hline & $55-3 \mathrm{C}$ & $\mathrm{Y}$ & & $\mathrm{Y}$ & & SID 2216 & D039, D040, D043 \\
\hline & $56-1 \mathrm{~A}$ & $\mathrm{Y}$ & & & & Not Contained &. \\
\hline & $56-1 \mathrm{C}$ & $\mathrm{Y}$ & & & & Not Contained & 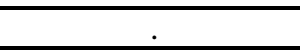 \\
\hline & $56-2 \mathrm{~A}$ & $\mathrm{Y}$ & & & & SID 2212 & \\
\hline & $56-2 \mathrm{~B}$ & $\mathrm{Y}$ & & & & SID 2216 & D039 \\
\hline & $56-2 C$ & $\mathrm{Y}$ & & & & SID 2216 & D039, D040 \\
\hline
\end{tabular}


Table F.2. (continued)

\begin{tabular}{|c|c|c|c|c|c|c|c|}
\hline \multirow{2}{*}{ Regime } & \multirow{2}{*}{ Location } & \multicolumn{4}{|c|}{ CY 2010 Sampling Qtr. } & \multirow{2}{*}{$\begin{array}{c}\text { Waste Stream ID (SID } \\
\#)\end{array}$} & \multirow{2}{*}{ RCRA Waste Code } \\
\hline & & Q1 & $\mathbf{Q 2}$ & Q3 & Q4 & & \\
\hline \multirow{50}{*}{$\begin{array}{l}\text { East Fork } \\
\text { (continued) }\end{array}$} & $56-3 \mathrm{~A}$ & $\bar{Y}$ & & & & SID 2212 & . \\
\hline & $56-3 \mathrm{~B}$ & $\bar{Y}$ & & & & SID 2212 & . \\
\hline & $56-3 C$ & $\bar{Y}$ & & & & SID 2212 & . \\
\hline & $56-4 \mathrm{~A}$ & $\bar{Y}$ & & & & SID 2212 & 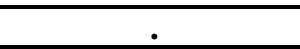 \\
\hline & $56-6 \mathrm{~A}$ & & & & $\mathrm{Y}$ & Not Contained &. \\
\hline & $56-8 \mathrm{~A}$ & $\bar{Y}$ & & & & SID 2212 & . \\
\hline & $60-1 \mathrm{~A}$ & & $\mathrm{Y}$ & & & Not Contained & $\dot{.}$ \\
\hline & GW-106 & & & & $\mathrm{Y}$ & SID 2212 & \\
\hline & GW-109 & & & & $\bar{Y}$ & SID 2216 & D005, D006 \\
\hline & GW-148 & $\bar{Y}$ & & & & SID 2212 & 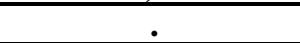 \\
\hline & GW-153 & & $\mathrm{Y}$ & & & SID 2212 & . \\
\hline & GW-204 & & & & $\mathrm{Y}$ & SID 2212 & \\
\hline & GW-220 & $\bar{Y}$ & & $\bar{Y}$ & & SID 2216 & D019 \\
\hline & GW-240 & & $\mathrm{Y}$ & & & SID 2212 & 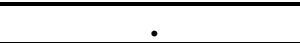 \\
\hline & GW-251 & & & & $\mathrm{Y}$ & SID 2212 & $\dot{.}$ \\
\hline & GW-269 & & & & $\mathrm{Y}$ & SID 2212 & \\
\hline & GW-274 & & & & $\bar{Y}$ & SID 2216 & D039 \\
\hline & GW-275 & & & & $\bar{Y}$ & SID 2216 & D005 \\
\hline & GW-332 & & & & $\mathrm{Y}$ & SID 2216 & D039 \\
\hline & GW-337 & & & & $\mathrm{Y}$ & SID 2216 & D039, D040 \\
\hline & GW-381 & & $\mathrm{Y}$ & & & SID 2212 &. \\
\hline & GW-383 & & $\mathrm{Y}$ & & 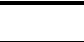 & SID 2212 & . \\
\hline & GW-619 & & $\mathrm{Y}$ & & 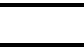 & SID 2212 & . \\
\hline & GW-620 & & $\mathrm{Y}$ & & & Not Contained & \\
\hline & GW-633 & & & & $\bar{Y}$ & SID 2216 & $\begin{array}{l}\text { D018 } \\
\end{array}$ \\
\hline & GW-656 & & & & $\mathrm{Y}$ & SID 2216 & D040 \\
\hline & GW-686 & & & & $\mathrm{Y}$ & SID 2212 & \\
\hline & GW-690 & & & & $\mathrm{Y}$ & SID 2212 & \\
\hline & GW-691 & & $\mathrm{Y}$ & & $\bar{Y}$ & SID 2216 & D039 \\
\hline & GW-692 & & & & $\overline{\mathrm{Y}}$ & SID 2212 & \\
\hline & GW-698 & & $\mathrm{Y}$ & & $\mathrm{Y}$ & SID 2216 & D040 \\
\hline & GW-700 & $\bar{Y}$ & & & & SID 2212 & . \\
\hline & GW-722-14 & & & $\bar{Y}$ & & SID 2212 & . \\
\hline & GW-722-17 & & & $\mathrm{Y}$ & & SID 2212 & - \\
\hline & GW-722-20 & & & $\mathrm{Y}$ & & SID 2212 & 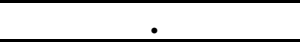 \\
\hline & GW-722-22 & & & $\mathrm{Y}$ & & SID 2212 & . \\
\hline & GW-722-33 & & & $\bar{Y}$ & & Not Contained & . \\
\hline & GW-744 & & & $\mathrm{Y}$ & & Not Contained & . \\
\hline & GW-747 & & & $\mathrm{Y}$ & & Not Contained & . \\
\hline & GW-748 & & & $\mathrm{Y}$ & & Not Contained & . \\
\hline & GW-763 & & $\mathrm{Y}$ & & & Not Contained & . \\
\hline & GW-769 & & $\bar{Y}$ & & $\overline{\mathrm{Y}}$ & SID 2212 & 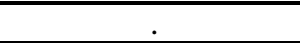 \\
\hline & GW-770 & & $\mathrm{Y}$ & & & SID 2212 & 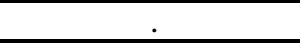 \\
\hline & GW-781 & $\mathrm{Y}$ & & & & Not Contained & . \\
\hline & GW-782 & $\mathrm{Y}$ & & & & SID 2212 & . \\
\hline & GW-783 & $\mathrm{Y}$ & & & & SID 2212 & \\
\hline & GW-791 & $\mathrm{Y}$ & & & & SID 2212 & \\
\hline & GW-792 & $\mathrm{Y}$ & & & & Not Contained & \\
\hline & GW-816 & $\mathrm{Y}$ & & & & Not Contained & \\
\hline & GW-820 & & & & $\bar{Y}$ & SID 2216 & D039, D040 \\
\hline
\end{tabular}




\section{DISTRIBUTION}

\section{U.S. DEPARTMENT OF ENERGY}

J. P. Donnelly *

ENVIRONMENTAL COMPLIANCE DEPARTMENT

S. M. Field

C. C. Hill

S. B. Jones *

S. Jollay *

B. E. Skaggs

L. O. Vaughan

ANALYTICAL CHEMISTRY ORGANIZATION

M.C. Tucker-Watson

INFORMATION TECHNOLOGY

S. W. King

Y-12 Central Files *

9114DMC-01971865.6550-RC

Y-12 Records Services (Electronic copy- OSTI)

YDCC - RC *
TENNESSEE DEPARTMENT OF

ENVIRONMENT AND CONSERVATION

DOE OVERSIGHT

J. E. Sebastian *

ELVADO ENVIRONMENTAL LLC

T. R. Harrison *

BECHTEL JACOBS COMPANY LLC

E. L. Berglund *

H. K. Haase

R. H. Ketelle *

L. M. Sims *

File-EMEF-DMC *

File-Y-12 Project PDCC *

COMMODORE ADVANCED SCIENCES, INC.

M. Selecman

UT-BATTELLE, LLC

D. B. Watson

Note: $*$ = receives hard copy version 